\title{
Teoremi di confronto per i sistemi di equazioni differenziali ordinarie e loro conseguenze $\left({ }^{*}\right)$.
}

\author{
Memoria di Mauro Picone (a Roma).
}

Sunto. - Si danno alcuni teoremi di confronto per sistemi di equazioni differenziali ordinarie e se ne fa applicazione ad wha vasta classe di problemi di integrazione di sistemi di equazioni lineari differenziali ordinarie con condizioni non iniziali (integrali o assegnanti alle solueioni valori in determinati punti), nonchè allo studio della dipendenza delle soluzioni dei sistemi non lineari, da un parametro che compare nelle equazioni $e$ nelle condizioni iniziali.

Nella Memoria di CaRLo MrRanda dedicata alle ricerche compiute, fra il 1932-XI e il 1933-XII, nell' Istituto Nazionale per le Applicazioni del Calcolo, concernenti i problemi che si riallaciano all' integrazione dell' equazione differenziale Thomas-Ferm della Fisica atomica ( $\left.{ }^{1}\right)$, trovasi enunciato $\left({ }^{2}\right)$ senza dimostrazione, e utilizzato un generale teorema di confronto, fra equazioni differenziali ordinarie del secondo ordine, che io ebbi occasione di suggexire in quelle ricerche ai miei collaboratori dell' Istituto, e al quale oggi, antorevolmente sollecitato a farne conoscere la dimostrazione, ritengo utile destinare la presente trattazione che lo fa discendere, immediatamente ed in ipotesi molto più larghe di quelle allora considerate, da taluni generali teoremi di confronto relativi a sistemi di quante si vogliano equazioni differenziali ordinarie, in altrettante funzioni incognite, i quali teoremi, inoltre, come pure mostrero nel presente scritto, conducono ad uno nuovo esistenziale per gl'integrali dei sistemi di equazioni differenziali ordinarie, dipendenti da un parametro, valevole, a differenza dei classici teoremi, in grande nel parametro, valevole cioè al variare di questo in un prefissato intervallo, e, per ora limitatamente ai sistemi lineari, forniscono larga messe di notevoli criteri sufficienti d'esistenza per le soluzioni assoggettate a ulteriori equazioni lineari non differenziali di varii tipi.

(*) Lavoro eseguito nell' Istituto Narionale per le Applicazioni del Calcolo.

(1) C. Miranda, Teoremi e metodi per l'integrazione numerica della equazione differen. ziale di Fermi, [" Memorie della Reale Accademia d' Italia ", Vol. V (1934-XII)], pp. 285-322. (2) A pag. 291. 
È opportunissimo, anche per le concrete applicazioni $\left({ }^{3}\right)$, considerare i detti sistemi di equazioni differenziali, nelle ipotesi sulle quali il Caratheo. DORY ( $\left.{ }^{4}\right)$ ne ha costruito una ben nota fruttuosa teoria e pertanto, per ben intendere ed evitare noiose ripetizioni, mi permetto di introdurre qui talune locuzioni che vado, come prima cosa, a definire.

Indichino $x, y_{1}, \ldots, y_{p}$ quantità variabili reali indipendenti e $R$ il dominio rettangolare definito dalle limitazioni

$$
a \leqq x \leqq b, \quad a_{h} \leqq y_{h} \leqq b_{h} \quad(h=1, \ldots, p),
$$

cioè, come diremo, di punto estremo inferiore $\left(a, a_{1}, \ldots, a_{p}\right)$ e superiore $\left(b, b_{1}, \ldots, b_{p}\right)$. Diremo che una funzione reale

$$
f\left(x, y_{1}, \ldots, y_{p}\right)
$$

delle variabili reali $x, y_{1}, \ldots, y_{p}$, è, nel dominio $R$, del tipo di Caratheodory, rispetto alla variabile $\mathrm{x}, 0$, brevemente, del tipo $\mathrm{C}$, rispetto alla $\mathrm{x}$, se:

$\left.1^{\circ}\right)$ per un certo insieme $N$ di punti dell' intervallo $(a, b)$, eventualmente anche vuoto, di misura (lebesguiana) nulla, è ben definita per ogni punto $\left(x, y_{1}, \ldots, y_{p}\right)$ di $R$, per il quale $x$ non appartenga ad $N$;

$\left.2^{\circ}\right)$ comunque si fissi $x$ in $(a, b)$, fuori di $N$, la $f$ è funzione continua del punto $\left(y_{1}, \ldots, y_{p}\right)$ nel dominio rettangolare, dello spazio a $p$ dimensioni, di punto estremo inferiore $\left(a_{1}, \ldots, a_{p}\right)$ e superiore $\left(b_{1}, \ldots, b_{p}\right)$ e comunque si fissi $\left(y_{1}, \ldots, y_{p}\right)$ in tale dominio $\mathrm{e}$ in $(a, b)$ funzione misurabile di $x$;

$3^{\circ}$ ) esiste una funzione $F(x)$, dell' unica variabile $x$; sommabile in $(a, b)$, per la quale, in $R$, risulta

$$
\left|f\left(x, y_{1}, \ldots, y_{p}\right)\right| \leqq F(x) .
$$

Diremo che la funzione $f \grave{e}$, nel dominio rettangolare $R$, generalmente continua, rispetto alla $\mathrm{x}$, se:

per un certo insieme $N$ di punti dell' intervallo $(a, b)$, eventualmente anche vuoto, privo di derivato, la $f$ è ben definita in ogni punto $\left(x, y_{1}, \ldots, y_{p}\right)$ di $R$ per il quale $x$ non appartenga ad $R$, e vi è continma come funzione del punto $\left(x, y_{1}, \ldots, y_{p}\right)$.

(3) Anche, per esempio, per la ricordata equazione Thomas-Ferma:

$$
y^{\prime \prime}=\frac{y^{\frac{3}{2}}}{\sqrt{x}}
$$

che deve considerarsi nell' intervallo $(0, \infty)$.

(i) O. CARATHeodory, Vorlesungen über reelle Funktionen, [B. G. Teubner (1918 e 1927)], pp. $665-688$. 
Ovviamento, se la $f$ è lineare rispetto alle $y_{1}, \ldots, y_{p}$, ed è

$$
f=\varphi(x)+\sum_{h=1}^{p} \varphi_{h}(x) y_{h},
$$

la $f$ riesce, nel dominio $R$, del tipo $C$ rispetto alla $x$, non appena le funzioni $\varphi, \varphi_{1}, \ldots, \varphi_{p}$, della $x$, sono sommabili in $(a, b)$ e gl' intervalli $\left(a_{h}, b_{h}\right)$ $(h=1, \ldots, p)$ sono finiti. Nelle stesse ipotesi, se le funzioni $\varphi, \varphi_{1}, \ldots, \varphi_{p}$ sono continue in ogni punte di $(a, b)$, fuori di un certo insieme $N$ privo di derivato, la $f \dot{e}$ anche generalmente continua, rispetto alla $x$, nel dominio $R$.

Diremo che il sistema di funzioni rẹali $y_{1}(x), y_{2}(x), \ldots, y_{p}(x)$, che indiche. remo con $\left[y_{h}(x)\right]$ e, in $R$, soluzione del sistema di equazioni differenziali

$$
y_{n}{ }^{\prime}=f_{n}\left(x, y_{1}, \ldots, y_{p}\right), \quad(h=1, \ldots, p),
$$

se le dette funzioni, in ogni intervallo finito di $(a, b)$, sono assolutamente continue, verifieano, in $(a, b)$, le limitazioni aperte

$$
a_{h}<y_{h}(x)<b_{h}, \quad(h=1, \ldots, p),
$$

ed infine, con eccezione, al più, dei punti di $(a, b)$ di un insieme di misura nullo, soddisfano le equazioni.

Così, data in $R$ una funzione $f\left(x, y_{1}, \ldots, y_{p}\right)$ del tipo $C$ rispetto alla $x$, diremo che la funzione reale $y(x)$, è, in $R$, soluzione dell' equazione differen. ziale d'ordine $p$ :

$$
y^{(p)}=f\left(x, y, y^{\prime}, \ldots, y^{(p-1)}\right),
$$

se la funzione e tutte le sue derivate, fino a quella inclusa d'ordine $p-1$, sono assolutamente. continue in ogni intervallo finito di $(a, b)$, verificano, in $(a, b)$, le limitazioni aperte

$$
a_{1+k}<y^{(k)}(x)<b_{1+k}, \quad(k=0.1, \ldots, p-1),
$$

ed infíne, con eccezione, al più, dei punti di $(a, b)$ di un insieme di misurá nulla, soddisfano l'equazione.

Il sistema di equazioni differenziali (1), avente, in $R$, la soluzione $\left[y_{h}(x)\right]$, si dirà unitario in tale soluzione, se, per ogni altra soluzione $\left[Y_{h}(x)\right]$, in $R$, si ha, in $(a, b)$,

$$
Y_{h}(x) \equiv y_{h}(x), \quad(h=1, \ldots, p),
$$

non appena, per un punto $x_{0}$ di $(a, b)$, si abbia

$$
Y_{h}\left(x_{0}\right)=y_{h}\left(x_{0}\right), \quad(h=1, \ldots, p) ;
$$

il sistema si dirà unitario, in $\mathrm{R}$, se è tale in ogni sua soluzione, in $R$. 
Così l'equazione differenziale (2), avente, in $R$, la solnzione $y(x)$, si dirà unitaria in tale soluzione, se, per ogni altra soluzione $Y(x)$, in $R$, si ha, in $(a, b)$,

$$
Y(x) \equiv y(x)
$$

non appena, per un punto $x_{0}$ di $(a, b)$, si abbia

$$
Y^{(k)}\left(x_{0}\right)=y^{(k)}\left(x_{0}\right), \quad(k=0,1, \ldots, p-1) .
$$

Ciò posto, possiamo in modo breve enunciare un teorema, del quale faremo uso sovente, contenuto in uno dato dal CARatheodory nel luogo eitato $\left({ }^{5}\right)$, concernente l'integrazionè del sistema di equazioni differenziali $\theta$ di condizioni iniziali:

$$
\left\{\begin{array}{l}
y_{h}{ }^{\prime}=f_{h}\left(x, y_{1}, \ldots, y_{p}, \lambda\right), \\
y_{h}\left(x_{0}\right)=\alpha(\lambda),
\end{array} \quad(h=1, \ldots, p)\right.
$$

dipendente dal parametro $\lambda$.

I. Se nel dominio rettangolare, dello spazio $\left(\mathrm{x}, \mathrm{y}_{1}, \ldots, \mathrm{y}_{\mathrm{p}}, \lambda\right)$. di punto estremo inferiore $\left(a, a_{1}, \ldots, a_{p}, \lambda^{\prime}\right)$ e superiore $\left(b, b_{1}, \ldots, b_{p}, \lambda^{\prime \prime}\right)$, le funzioni $\mathrm{f}_{\mathrm{h}}$ $e \alpha_{\mathrm{h}}$ sono del tipo $\mathrm{C}$, rispetto alla $\mathrm{x}$, verificano le timitazioni

$$
a_{h}<\alpha_{h}(\lambda)<b_{h}, \quad(h=1, \ldots, p),
$$

$e$, per wn valore $\lambda_{0}$ interno all' intervallo $\left(\lambda^{\prime}, \lambda^{\prime \prime}\right)$ il sistema di equazioni differenziale $\grave{e}$ unitario in una sua soluzione $\left[\mathrm{y}_{\mathrm{h}}{ }^{\circ}(\mathrm{x})\right]$, nel dominio rettangolare $\mathrm{R}\left[\left(\mathrm{a}, \mathrm{a}_{1}, \ldots, \mathrm{a}_{\mathrm{p}}\right) ;\left(\mathrm{b}, \mathrm{b}_{1}, \ldots, \mathrm{b}_{\mathrm{p}}\right)\right]$, allon $a$, supposto l' intervallo $(\mathrm{a}, \mathrm{b})$ finito, si pù̀ costruire un intorno $\left(\lambda_{0}-\sigma, \lambda_{0}+\sigma\right)$ del punto $\lambda_{0}$, e, per $\lambda$ in tale intorno, definire una soluzione $\left[\mathrm{y}_{\mathrm{h}}(\mathrm{x}, \lambda)\right]$, in $\mathrm{R}$, del sistema di equazioni (3), riuscendo le $\mathrm{y}_{\mathrm{h}}(\mathrm{x}, \lambda)$ funzioni continue del punto $(\mathrm{x} . \lambda)$ in ogni punto $\left(\mathrm{x}, \lambda_{0}\right)$.

Tale teorema, come tutti quelli fino ad oggi - a quanto io so - stabiliti al riguardo, è dunque da reputarsi in piccolo nel parametro ( $\left.{ }^{6}\right)$.

1. Teoremi di confronto con relazioni iniziali. - $\mathrm{E}$ subito dimostrato il seguente teorema:

II. Supposto a al finito ( $\left.{ }^{7}\right)$, le funzioni

$$
f_{h}\left(x, y_{1}, \ldots, y_{p}\right), \quad g_{h}\left(x, y_{1}, \ldots, y_{p}\right), \quad(h=1, \ldots, p),
$$

siano di tipo $\mathrm{C}$, rispetto alla $\mathrm{x}$, nel dominio rettangolare $\mathrm{R}\left[\left(\mathrm{a}, \mathrm{a}_{1}, \ldots, \mathrm{a}_{\mathrm{p}}\right)\right.$;

(5) A pag. 678 del luogo citato [loc. eit. $\left.\left.{ }^{4}\right)\right]$.

(6) Chr. anche E. KAMKE, Differentialgleichungen reelier Funltionen, [" Akademische Verlongsgesellschaft M. B. H. ", Leipzig (1980)], \$§ 10 e 17.

(7) E ciò sarà sempre sottinteso nel seguito. 
$\left.\left(\mathrm{b}, \mathrm{b}_{1}, \ldots, \mathrm{b}_{\mathrm{p}}\right)\right]$ e si pongano a confronto $i$ due sistemi

$$
\begin{array}{ll}
y_{h}{ }^{\prime}=f_{h}\left(x, y_{1}, \ldots, y_{p}\right), & (h=1, \ldots, p), \\
y_{h}{ }^{\prime}=g_{h}\left(x, y_{1}, \ldots, y_{p}\right) . & (h=1, \ldots, p),
\end{array}
$$

dei quali $\left[\mathrm{u}_{\mathrm{h}}(\mathrm{x})\right]$ e $\left[\mathrm{v}_{\mathrm{h}}(\mathrm{x})\right]$ sono, rispettivamente, soluzioni in $\mathrm{R}$.

Detto $\mathrm{N}$ un insieme, di punti di (a,b), di misura nulla, si verifichi una delle seguenti ipotesi :

a') essendo il sistema (5) unitario nella soluzione $\left.] \mathrm{V}_{\mathrm{h}}(\mathrm{x})\right]$, comunque si fissi $\mathrm{x}$ in (a, b), fuori di $\mathrm{N}$, le $\mathrm{g}_{\mathrm{h}}$ sono funzioni non decrescenti di ciascuna delle rimanenti variabili, ed è

$$
f_{h}\left(x, u_{1}(x), \ldots, u_{p}(x)\right) \leqq g_{h}\left(x, u_{1}(x), \ldots, u_{p}(x)\right), \quad(h=1, \ldots, p) ;
$$

$\left.\mathrm{a}^{\prime \prime}\right)$ essendo il sistema (4) unitario nella soluzione $\left[\mathrm{u}_{\mathrm{n}}(\mathrm{x})\right]$, comunque si fissi $\mathrm{x}$ in $(\mathrm{a}, \mathrm{b})$, fuori di $\mathrm{N}$, le $\mathrm{f}_{\mathrm{h}}$ sono funzioni non decrescenti di ciascuna delle rimanenti variabili, ed è

$$
f_{h}\left(x, v_{1}(x), \ldots, v_{p}(x)\right) \leqq g_{h}\left(x, v_{1}(x), \ldots, v_{p}(x)\right), \quad(h=1 \ldots, p),
$$

allora, dalle relazioni iniziali

$$
u_{h}(a) \leqq v_{h}(a), \quad(h=1, \ldots, p),
$$

si deducono, in tutto $(\mathrm{a}, \mathrm{b})$, le seguenti

$$
u_{h}(x) \leqq v_{h}(x)
$$$$
(h=1, \ldots, p)
$$

Mettiamoci, per esempio, nell' ipotesi a') e supponiamo, dapprima, che si abbia

$$
u_{h}(a)<v_{h}(a), \quad(h=1, \ldots, p) .
$$

Dico ehe allora sarà in tutto $(a, b)$

$$
u_{h}(x)<v_{h}(x), \quad(h=1, \ldots, p) .
$$

Ed invero se le (9) non avessero luogo in tutto $(a, b)$, esisterebbero un indice $i$ e un punto $\xi>a$, di $(a, b)$, per i quali risulterebbe

Ma si ha

$$
\begin{cases}v_{h}(x)-u_{h}(x)>0, \text { per } a \leqq x<\xi, & (h=1, \ldots, p), \\ v_{h}(\xi)-u_{h}(\xi) \geqq 0, & (h=1, \ldots, p), \\ v_{i}(\xi)-u_{i}(\xi)=0, & \end{cases}
$$

$$
\begin{aligned}
v_{i}(\xi)-u_{i}(\xi) & =v_{i}(a)-u_{i}(a)+\int_{a}^{\xi}\left[g_{i}\left(x, v_{1}(x), \ldots, v_{p}(x)\right)-f_{i}\left(x, u_{1}(x), \ldots, u_{p}(x)\right)\right] d x \\
& \geqq v_{i}(a)-u_{i}(a)+\int_{a}^{\xi}\left[g_{i}\left(x, u_{i}(x), \ldots, u_{p}(x)\right)-f_{i}\left(x, u_{1}(x), \ldots, u_{p}(x)\right)\right] d x \\
& \geqq v_{i}(a)-u_{i}(a)>0,
\end{aligned}
$$


onde l'assurdo. Sussistano ora le (7). Introdotto an parametro reale $\lambda$, consideriamo il sistema (5) con le condizioni iniziali

$$
y_{h}(a)=v_{h}(a)+\lambda, \quad(h=1, \ldots, p) .
$$

Comunque si fissi una quantità finita $\xi \leqq b$, per il teor. I. di CaratheoDORY si può determinare un numero positivo $\sigma$ tale che, per $|\lambda|<\sigma$, esiste, nel dominio rettangolare $R_{\xi}\left[\left(a, a_{1}, \ldots, a_{p}\right) ;\left(\xi, b_{1}, \ldots, b_{p}\right)\right]$. una soluzione $\left[V_{h}(x, \lambda)\right]$ del sistema (5) con le condizioni iniziali (11), essendo le $V_{h}(x, \lambda)$ funzioni continue del punto $(x, \lambda)$, in ogni punto $(x, 0)$, per $x$ nell' intervallo $(a, \xi)$. Ma, per quanto abbiamo già dimosrrato, si ha, in $(\alpha, \xi)$,

donde

$$
V_{h}(x, \lambda)>u_{h}(x), \quad \text { per } \lambda>0, \quad(h=1, \ldots, p),
$$

$$
v_{h}(x)=\lim _{\lambda \rightarrow 0} V_{h}(x, \lambda)(\text { per } \lambda>0) \geqq u_{h}(x), \quad(h=1, \ldots, p)\left({ }^{8}\right) .
$$

Dimostrazione di pari semplicità ha il seguente ulteriore teorema di confronto:

III. Le funzioni

$$
\begin{aligned}
f_{h} & \equiv \omega_{h}(x) \varphi_{h}\left(x, y_{1}, \ldots, y_{p}\right), \\
g_{h} & \equiv \omega_{h}(x) \psi_{h}\left(x, y_{1}, \ldots, y_{y}\right),
\end{aligned} \quad(h=1, \ldots, p)
$$

siano, rispetto alla $\mathrm{x}$, del tipo $\mathrm{O}$ nel dominio $\mathrm{R}$, le $\varphi_{\mathrm{h}} e \psi_{\mathrm{h}}$ generalmente continue, le $\omega_{\mathrm{h}}(\mathrm{x})$, dipendenti soltanto dalla $\mathrm{x}$, sommabili in $(\mathrm{a}, \mathrm{b})$, e ivi quasi

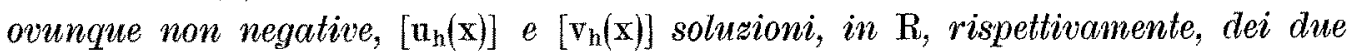
sistemi

$$
\begin{array}{ll}
y_{h}{ }^{\prime}=\omega_{h}(x) \varphi_{h}\left(x, y_{1}, \ldots, y_{p}\right), & (h=1, \ldots, p) \\
y_{h}{ }^{\prime}=\omega_{h}(x) \psi_{h}\left(x, y_{1}, \ldots, y_{p}\right), & (h=1, \ldots, p) .
\end{array}
$$

Detto $\mathrm{N}$ un insieme di punti di (a, b), privo di derivato, la $\varphi_{\mathrm{h}} e \psi_{\mathrm{h}}$ siano,

(8) Dunque, in particolare, dal confronto dei due sistemi:

$$
\begin{aligned}
& y_{h}^{\prime}=0 \\
& y_{h}{ }^{\prime}=g_{h}\left(x, y_{1}, \ldots, y_{p}\right),
\end{aligned}
$$

essendo le $g_{h}$ del tipo $c$, rispetto alla $x$, e il sistema (5) unitario in una sua soluzione $\left[v_{h}(x)\right]$, se, comunque si fissi $x$ in $(a, b)$, fuori di un certo insieme di misura nulla, le $g_{h}$ sono funzioni non decrescenti di ciascuna delle rimanenti variabili, ed esistono $p$ costanti $c_{1}, \ldots, c_{p}$ interne, rispettivamente, agli intervalli $\left(a_{1}, b_{1}\right), \ldots,\left(a_{p}, b_{p}\right)$, per le quali riesce, quasi ovunque in $(a, h)$,

dalle relazioni iniziali

$$
y_{h}\left(x, c_{1}, \ldots, c_{p}\right) \geqq 0, \quad(h=1, \ldots, p),
$$

si deducono le seguenti, in tutto $(a, b)$,

$$
v_{l}(a) \geqq c_{l},
$$$$
(h=1, \ldots, p) \text {, }
$$

$v_{h}(x) \geqq c_{h}$

$(h=1, \ldots, p)$. 
in $\mathrm{R}$, funzioni continue del punto $\left(\mathrm{x}, \mathrm{y}_{1}, \ldots, \mathrm{y}_{\mathrm{p}}\right)$, per $\mathrm{x}$ fuori di $\mathrm{N}$ e si verifichi una delle seguenti ipotesi:

$\left.b^{\prime}\right)$ essendo il sistema (13) unitario nella soluzione $\left[\mathrm{v}_{\mathrm{h}}(\mathrm{x})\right]$, comunque si fissino $\mathrm{x}$ in $(\mathrm{a}, \mathrm{b})$, fuori $d i \mathrm{~N}, \quad e \mathrm{y}_{\mathrm{h}}$, interno all'intervallo $\left(\mathrm{a}_{\mathrm{h}}, \mathrm{b}_{1}\right), \quad$ a $\psi_{\mathrm{h}}(\mathrm{h}=1, \ldots, \mathrm{p})$ è fuuzione non decrescente di ciascuna delle rimanenti varicibili $\mathrm{y}_{1}, \ldots, \mathrm{y}_{\mathrm{h}-1}, \mathrm{y}_{\mathrm{h}+1}, \ldots, \mathrm{y}_{\mathrm{p}}$ ed $\grave{e}$

$$
\varphi_{h}\left(x, u_{1}(x), \ldots, u_{p}(x)\right) \leqq \Psi_{h}\left(x, u_{1}(x), \ldots, u_{p}(x)\right), \quad(h=1, \ldots, p) ;
$$

$\left.b^{\prime \prime}\right)$ essendo il sistema (12) unitario nella soluzione $\left[\mathrm{u}_{\mathrm{h}}(\mathrm{x})\right]$, comunque si fissino $\mathrm{x}$ in $(\mathrm{a}, \mathrm{b})$, fuori di $\mathrm{N}, e \mathrm{y}_{\mathrm{h}}$ interno, all'intervallo $\left(\mathrm{a}_{\mathrm{h}}, \mathrm{b}_{\mathrm{h}}\right)$, la $\varphi_{\mathrm{h}}(\mathrm{h}=1, \ldots, \mathrm{p}) \dot{e}$ funsione non decresente di ciascuna delle rimaneni $v a$ riabili $\mathrm{y}_{1}, \ldots, \mathrm{y}_{\mathrm{h}-1}, \mathrm{y}_{\mathrm{h}+1}, \mathrm{y}_{\mathrm{p}}, e d \grave{e}$

$$
\varphi_{h}\left(x, v_{1}(x), \ldots, v_{p}(x)\right) \leqq \psi_{h}\left(x, v(x), \ldots, v_{p}(x)\right), \quad(h=1, \ldots, p) ;
$$

allora dalle relazioni iniziali (7) si deducono, in tulto (a, b), le (8).

Ordinati i punti di $N$, distinti da $a$, secondo la successione crescente

$$
x_{1}, x_{2}, \ldots, x_{n}, \ldots,
$$

e dimostrate, con un certo ragionamento, le (8) per ogni punto interno al. l'intervallo $\left(a, x_{1}\right)$, ne seguirà $u_{h}(x) \leqq v_{h}(x)$. Sostituito $a$ con $x_{1}$, mediante lo stesso ragionamento si dimostreranno le (8) per ogni punto interno all'intervallo $\left(x_{1}, x_{2}\right)$ e ne seguirà $u_{h}\left(x_{2}\right) \leqq v_{h}\left(x_{2}\right)$, ece. Tutto dunque si riduce a esporre il ragionamento che assicuri la validita delle (8) per ogni punto interno all'intervallo $\left(a, x_{1}\right)$. Mettiamoci; per esempio, nell' ipotesi $\left.b^{\prime}\right)$ e supponiamo, dapprima, che si abbia

e, per $a<x<x_{1}$

$$
u_{h}(a)<v_{h}(a), \quad(h=1, \ldots, p),
$$

$$
\varphi_{h}\left(x, u_{1}(x), \ldots, u_{p}(x)\right)<\psi_{h}\left(x, u_{1}(x), \ldots, u_{p}(x)\right) .
$$

Dico ehe, allora, varranno le $(9)$, per $a<x<x_{1}$. Ed invero, se ciò non avesse linogo, esisterebbero un punto $\xi$ interno all'intervallo $\left(a, x_{1}\right)$ ed un indice $i$ per i quali si verificherebbero le (10), e quindi, detto $c$ il valore comune di $u_{2}(\xi)$ e $v_{1}(\xi)$

$$
\begin{gathered}
\psi_{i}\left(\xi, v,(\xi), \ldots, v_{p}(\xi)\right)-\varphi_{1}\left(\xi, u_{1}(\xi), \ldots, u_{p}(\xi)\right)= \\
=\psi_{1}\left(\xi, \ldots, v_{2-1}(\xi), c, v_{i+1}(\xi), \ldots\right)-\varphi_{i}\left(\xi, \ldots, u_{i-1}(\xi), c, u_{i+1}(\xi), \ldots\right) \geqq \\
\geqq \psi_{i}\left(\xi, \ldots, u_{i-1}(\xi), c, u_{i+1}(\xi), \ldots\right)-\varphi_{i}\left(\xi, \ldots, u_{i-1}(\xi), c, u_{i+1}(\xi), \ldots\right)>0 .
\end{gathered}
$$

Ne segue, per la supposta continuita delle funzioni $\varphi$ e $\psi$. l'esistenza di un punto $\alpha$, interno all'intervallo $(\alpha, \xi)$ tale che, nell'intervallo $(\alpha, \xi)$, si ha 
sempre

$$
\psi_{\imath}\left(x, v_{1}(x), \ldots, v_{p}(x)\right) \rightarrow \varphi_{i}\left(x, u_{1}(x), \ldots, u_{p}(x)\right)>0
$$

e pertanto

$$
\begin{aligned}
& v_{1}(\xi)-u_{1}(\xi)= \\
& =v_{i}(\alpha)-u_{i}(\alpha)+\int_{\alpha}^{\sum_{\alpha}}\left[\psi\left(x, v_{1}(x), \ldots, v_{p}(x)\right)-\varphi_{i}\left(x, u_{1}(x), \ldots, u_{p}(x)\right)\right] \omega,(x) d x \geqq \\
& \geqq v_{i}(\alpha)-u_{i}(\alpha)>0 \text {, }
\end{aligned}
$$

onde l'assurdo. Sussistano ora le relazioni delle ipotesi del teorema. In trodotto un parametro $\lambda$, consideriamo il seguente sistema di equazioni differenziali e di condizioni iniziali

$$
\left\{\begin{array}{l}
y_{h}^{\prime}=\left[\psi_{h}\left(x, y_{1}, \ldots, y_{p}\right)+\lambda\right] \omega_{h}(x), \\
y_{h}(\alpha)=v_{h}(a)+\lambda
\end{array} \quad(h=1, \ldots, p)\right.
$$

Comunque si fissi un punto $\xi$, interno all' intervallo $\left(a, x_{1}\right)$, si può determinare un numero positivo $\sigma$ tale che, per $|\lambda|<\sigma$, esiste una soluzione $\left[V_{h}(x, \lambda)\right]$, nel dominio rettangolare $R_{\xi}$, del sistema $(14)$, essendo le $V_{h}(x, \lambda)$ funzioni continue del punto $(x, \lambda)$, in ogni punto $(x, 0)$, per $x$ in $(a, \xi)$. Ma, per quanto abbiamo già dimostrato, si ha, in $(a, \xi)$,

donde

$$
V_{h}(x, \lambda)>v_{h}(x), \quad \text { per } \lambda>0, \quad(h=1, \ldots, p),
$$

$$
v_{h}(x)=\lim _{\lambda \rightarrow 0} V_{h}(x, \lambda)(\text { per } \lambda>0) \geqq u_{h}(x), \quad(h=1, \ldots, p) \quad\left({ }^{9}\right)
$$

Conviene esplicitamente enunciare il caso particolare per $p=1$ del teorema ora dimostrato.

$\left({ }^{9}\right)$ In particolare, dal confronto dei due sistemi:

$$
\begin{aligned}
& y_{h}{ }^{\prime}=0 \\
& y_{h}{ }^{\prime}=\omega_{h}(x) \psi_{h}\left(x, y_{1}, \ldots, y_{p}\right)
\end{aligned}
$$

essendo, rispetto alla $x$, le $\omega_{h} \psi_{h}$ del tipo $C$, le. $\psi_{h}$ generalmente continue, le $\omega_{h}(x)$ sommabili in $(a, b)$, ivi quasi ovunque non negative, il sistema (13) unitario in una sua soluzione $\left[v_{h}(x)\right]$, si deduce che: se, comunque si fissino $x$ in $(a, b)$, fnori di un certo insieme $N$ privo di derivato, o $y_{h}$ nell'interno dell'intervallo $\left(a_{h}, b_{h}\right)$, la $\psi_{h}(h=1, \ldots, p)$ è funzione non decrescente di ciascuna delle rimanenti variabili, ed esistono $p$ costanti $c_{1}, \ldots, c_{p}$, rispetivamente interne agli intervalli $\left(a_{1}, b_{1}\right), \ldots,\left(a_{p}, b_{p}\right)$, per le quali riesce, fuori di $N$,

dalle relazioni iniziali

$$
\Psi_{h}\left(x, c_{1}, \ldots, c_{p}\right) \geq 0, \quad(h=1, \ldots, p),
$$

si deducono le seguenti, in tutto $(a, b)$,

$$
v_{h}(a) \geqq c_{h}
$$

$$
v_{h}(x) \geq c_{n}
$$


IV. Le funzioni

$$
f \equiv \omega(x) \varphi(x, y), \quad g \equiv \omega(x) \psi(x, y),
$$

siano, rispetto alla $\mathrm{x}$, del tipo $\mathrm{C}$ nel dominio rettangolare $\mathrm{R}\left[\left(\mathrm{a}, \mathrm{a}_{4}\right) ;(\mathrm{b}, \mathrm{b}),\right]$, le $\varphi$ e $\psi$ generalmente continue, la $\omega(\mathrm{x})$ sommabile in $(\mathrm{a}, \mathrm{b})$, e ivi quasi ovunque non negativa, $\mathrm{u}(\mathrm{x})$ e $\mathrm{v}(\mathrm{x})$ soluzioni, in $\mathrm{R}$, rispettivamente, delle due equazioni

$$
\begin{aligned}
& y^{\prime}=\omega(x) \varphi(x, y), \\
& y^{\prime}=\omega(x) \psi(x, y) .
\end{aligned}
$$

Detto $\mathrm{N}$ un insieme di punti di (a, b), privo di derivata, le $\varphi$ e $\psi$ siano in $\mathrm{R}$ funzioni continue del pnnto $(\mathrm{x}, \mathrm{y})$, per $\mathrm{x}$ fuori $d i \mathrm{~N}$, e si verifichi una delle seguenti ipotesi:

$\left.\mathrm{c}^{\prime}\right)$ essendo la (16) unitaria nella soluzione $\mathrm{v}(\mathrm{x})$, comunque si fissi $\mathrm{x}$ fuori di $\mathrm{N}$, riesce

$$
\varphi(x, u(x)) \leqq \Psi(x, u(x))
$$

$\mathrm{c}^{\prime \prime}$ ) essendo la (15) unitaria nella soluzione $\mathrm{a}(\mathrm{x})$, comunque si fissi $\mathrm{x}$ fuori di $\mathrm{N}$, riesce

$$
\varphi(x, v(x)) \leqq \psi(x, v(x))
$$

allora, dalla relazione iniziale

$$
u(\alpha) \leqq v(\alpha)
$$

si deduce, in tutto (a, b), la seguente

$$
u(x) \leqq v(x) \quad{ }^{10} \%
$$

Il teor. II. fornisce, per le equazioni differenziali d'ordine $p, p \geqq 2$, il seguente che conviene enunciare.

V. Le funzioni

$$
f\left(x, y_{1}, \ldots, y_{p}\right), \quad g\left(x, y_{1}, \ldots, y_{p}\right),
$$

siano, rispetto alla $\mathrm{x}$, del tipo $\mathrm{O}$ nel dominio rettangolare $\mathrm{R}$, le $\mathrm{u}(\mathrm{x})$ e $\mathrm{v}(\mathrm{x})$, soluzioni, in $\mathrm{R}$, rispettivamente delle due equazioni

$$
\begin{aligned}
& y^{(p)}=f\left(x, y, y^{\prime}, \ldots, y^{(p-1)}\right), \\
& y^{(p)}=g\left(x, y, y^{\prime}, \ldots, y^{(p-1)}\right) .
\end{aligned}
$$

Detto $\mathrm{N}$ un insieme di punti di (a, b), di misura nulla, si verifichi una delle seguenti ipotesi:

$\left.\mathrm{a}^{\prime}\right)$ essendo l'equasione (18) unitaria nella soluzione $\mathrm{v}(\mathrm{x})$, comunque si fissi $\mathrm{x}$ in $(\mathrm{a}, \mathrm{b})$, fuori di $\mathrm{N}, l a \mathrm{~g}$ è funzione uon decrescente di ciascuna delle

(19) Teoremi analoghi al nostro teor. IV, in questo non contenuti e questo non contenenti, trovansi anche in KAMKE [loc. eit. $\left(^{6}\right)$, alle pp. 82 e 91. 
rimanenti variabili, ed è

$$
f\left(x, u(x), u^{\prime}(x), \ldots, u^{(p-1)}(x)\right) \leqq g\left(x, u(x), u^{\prime}(x) \ldots, u^{(p-1)}(x)\right) ;
$$

$\left.\mathrm{a}^{\prime \prime}\right)$ essendo l'equazione (17) unitaria nelln soluzione $\mathrm{u}(\mathrm{x})$, comunque si fissi $\mathrm{x}$ in (a, b), fuori di $\mathrm{N}$, la $\mathrm{f}$ è funzione non decrescente di ciascuna delle rimanenti variabili, ed $\grave{e}$

$$
f\left(x, v(x), v^{\prime}(x), \ldots, v^{(p-1)}(x)\right) \leqq g\left(x, v(x), v^{\prime}(x), \ldots, v^{(p-1)}(x)\right),
$$

allora dalle relazioni iniziali

$$
u^{(k)}(\alpha) \leqq v^{(k)}(\alpha), \quad(k=0,1, \ldots, p-1),
$$

si deducono, in tutto (a, b), le seguenti

$$
u^{(k)}(x) \leqq v^{(k)}(x), \quad(k=0,1, \ldots, p-1) .
$$

Interessa pare enunciare il seguente teorema fornito dal teor. III.

VI. Le funzioni

$$
f \equiv \omega(x) \varphi\left(x, y_{1}, \ldots, y_{p}\right), \quad g \equiv \omega(x) \psi\left(x, y_{1}, \ldots, y_{p}\right),
$$

siano, rispetto alla $\mathrm{x}$, del tipo $\mathrm{C}$ nel dominio reltangolare $\mathrm{R}$, le $\varphi$ e $\psi$ generalmente continue, $\omega(\mathrm{x})$ sommabile in $(\mathrm{a}, \mathrm{b})$, e ivi quasi ovwnque non negativa, le $\mathrm{u}(\mathrm{x})$ e $\mathrm{v}(\mathrm{x})$ soluzioni, in $\mathrm{R}$, rispettivamenle delle due equazioni

$$
\begin{aligned}
& y^{(p)}=\omega(x) \varphi\left(x, y, y^{\prime}, \ldots, y^{(p-1)}\right), \\
& y^{(p)}=\omega(x) \psi\left(x, y, y^{\prime}, \ldots, y^{(p-1)}\right) .
\end{aligned}
$$

Detto $\mathrm{N}$ un insieme di punti di (a, b), privo di derivato, le $\varphi$ e $\psi$ siano, in $\mathrm{R}$, funzioni continue del punto $\left(\mathrm{x}, \mathrm{y}_{1}, \ldots, \mathrm{y}_{\mathrm{p}}\right)$, per $\mathrm{x}$ fuori di $\mathrm{N}$, e si verifichi una delle seguenti ipotesi:

$\left.\mathrm{b}^{\prime}\right)$ essendo l'equazione (22) unitaria nella soluzione $\mathrm{v}(\mathrm{x})$, comunque si fissino $\mathrm{x}$ in $(\mathrm{a}, \mathrm{b})$, furi di $\mathrm{N}$, e $\mathrm{y}_{\mathrm{p}}$ interno all'intervallo $\left(\mathrm{a}_{\mathrm{p}}, \mathrm{b}_{\mathrm{p}}\right)$, le $\psi \dot{e}$ funzione non decrescente di ciascuna delle rimanenti variabili $\mathrm{y}_{1}, \mathrm{y}_{z}, \ldots, \mathrm{y}_{\mathrm{p}-1}$, ed è

$$
\varphi\left(x, u(x), u^{\prime}(x), \ldots, u^{(p-1)}(x)\right) \leqq \psi\left(x, u(x), u^{\prime}(x), \ldots, u^{(\tilde{E}-1)}(x)\right) ;
$$

$\mathrm{b}^{\prime \prime}$ ) essendo l'equazione (21) unitaria nella soluzione $\mathrm{u}(\mathrm{x})$, comunque si fissino $\mathrm{x}$ in (a, b), fuori di $\mathrm{N}$, e $\mathrm{y}_{\mathrm{p}}$ interno all' intervallo $\left(\mathrm{a}_{\mathrm{p}}, \mathrm{b}_{\mathrm{p}}\right)$, le $\varphi$ è funzione non decrescente di ciascuna delle rimanenti variabili, ed $\dot{e}$.

$$
p\left(x, v(x), v^{\prime}(x), \ldots, v^{(p-1)}(x)\right) \leqq \psi\left(x, v(x), v^{\prime}(x), \ldots, v^{(p-1)}(x)\right)
$$

allora dalle relazioni iniziali (19) si deducono in tutto (a, b), le (20) ${ }^{(11)}$.

(ii) Dnnque, in particolare, nelle ipotesi del teor. VI, dal confronto delle due equazioni:

$$
\begin{aligned}
& y^{(y)}=0 \\
& y^{(p)}=w(x) \psi\left(x, y, y^{\prime}, \ldots, y^{(p-1)}\right),
\end{aligned}
$$


2. Criteri suffeienti d'esistenza delle soluzioni per problemi d'integrazione di sistemi di equazioni differenziali lineari, con preseritte condizioni lineari. - Dal teorema osservato nella nota $\left({ }^{9}\right)$, per il sistema lineare

$$
y_{h}{ }^{\prime}=\varphi_{h}(x)+\sum_{k=1}^{p} \varphi_{h k}(x) y_{k}, \quad \quad(h=1, \ldots, p)
$$

con le $\varphi_{h}(x)$ e $\varphi_{h k}(x)$ generalmente continue in $(a, b)$ ed ivi sommabili, ponendovi $c_{3}=\ldots=c_{p}=\dot{0}$, si trae che, se generalmente,

$$
\varphi_{h}(x) \geqq 0, \quad \varphi_{h k}(x) \geqq 0, \quad(h \neq k, h, k=1, \ldots, p),
$$

una soluzione $\left[y_{h}(x)\right]$ per la quale ogni $y_{h}(x)$ è non negativa in $a$ tale si conserva in tutto $(a, b)$, e si avrà per tanto

cioè, posto

$$
y_{h}^{\prime}(x) \geq \varphi_{h}(x)+\varphi_{h h}(x) y_{h}
$$

si avrà, in $(a, b)$,

$$
y_{h}(\alpha)=\alpha_{h}, \quad \psi_{h}=y_{h}{ }^{\prime}-\varphi_{h}-\varphi_{h n} y_{h}
$$

$$
\begin{aligned}
& \psi_{n}(x) \geqq 0, \\
& y_{h}=\left(\alpha_{h}+\int_{a}^{\psi_{a}}\left[\varphi_{h}(\xi)+\psi_{h}(\xi)\right] e^{-\int_{a}^{\xi} \varphi_{h h}(t) d t} d \xi\right) e^{\int_{a}^{\infty} \varphi_{h, h}(\xi) d \xi},
\end{aligned}
$$

si deduce che, esiste un polinomio $n(x)$ di grado $p-1$, per il quale si ha, in $(a, b)$,

e fuori di $N$,

$$
a_{1+k}<u^{(k)}(x)<b_{1+k}, \quad(k=0,1, \ldots, p-1),
$$

$$
\psi\left(x, u(x), u^{\prime}(x), \ldots, u^{(p-1)}(x)\right) \geqq 0,
$$

dalle relazioni iniziali (19) seguono le (20). Si abbia, per esempio, l'equazione lineare

$$
y^{(p)}=\psi(x)+\psi_{0}(x) y+\psi_{1}(x) y^{\prime}+\ldots+\psi_{p-1}(x) y^{(p-1)},
$$

con $\psi, \psi_{0}, \ldots, \psi_{p-1}$ generalmente continue in $(\boldsymbol{a}, \boldsymbol{b})$, ed ivi sommabili, e sia, del pari generalmente,

$$
\psi_{0}(x) \geqq 0, \psi_{1}(x) \geq 0, \ldots, \psi_{p-2}(x) \geqq 0,
$$

$\psi_{p-1}(x)$ rimanendo non assoggettata ad aleuna condizione quantitativa, segue allora che, se, in $(a, b)$ è generalmente

$$
\psi(x) \geqq 0,
$$

uua soluzione dell' equazione non negativa in $a$, con tutte le sue prime $p-1$ derivate, si conserva tale in tutto $(a, b)$ : se, per una costante $e$, si ha generalmente

$$
\psi(x)+c \psi_{0}(x) \geq 0
$$

una soluzione dell'equazione in a non minore di $c$. le cui prime $p-1$ derivate sono in $a$ non negative si conserva tale in tutto $(a, b)$, ecc.. 
e quindi, con la limitazione, con secondo membro noto,

$$
y_{h}(x) \geqq\left(\alpha_{h}+\int_{a}^{\infty} \varphi_{h}(\xi) e^{-\int_{a} \varphi_{h h}(t) d t} d \xi\right) e^{\int_{a}^{x} \varphi_{h h}(\xi) d \xi},
$$

la proposizione fondamentale:

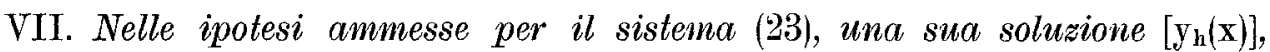
per la quale ogni $\mathrm{y}_{\mathrm{b}}(\mathrm{x})$ è non negativa in a, si conserva tale in tutto $(\mathrm{a} . \mathrm{b})$. Se, supposta b al finito, per un certo indice i riesce $\mathrm{y}_{\mathbf{i}}(\mathrm{b})=0$, si ha $\mathrm{y}_{\mathbf{i}}(\mathrm{x}) \equiv 0$ in tutto $(\mathrm{a}, \mathrm{b}) e$, in ogni punto di continuità delle $\varphi_{\mathrm{i}} e \varphi_{\mathrm{ih}}$,

$$
\varphi_{i}(\dot{x})=0, \quad \varphi_{i h}(x) y_{h}(x)=0, \quad(h=1, \ldots, p) .
$$

Supponiamo ora che, nelle dette ipotesi per il sistema (23), si abbia $y_{j}(a)>0$ e, per un certo indice $i$,

$$
y_{i}(a)=\alpha_{i}=0, \quad \varphi_{i}(x) \equiv 0,
$$

e ci domandiamo, supposto $b$ al finito, quando si potrà affermare che è $y_{i}(b)>0$ ? Si avrà sempre $y_{i}(b) \geqq 0$, e se fosse $y_{i}(b)=0$, si dedurrebbe $\varphi_{i, j}(x) y_{j}(x) \equiv 0$ e quindi $\varphi_{i j}(x) \equiv 0$ donde se non è $\varphi_{i, j}(x) \equiv 0$ dovrà risultare $y_{\imath}(b)>0$. Sia $\varphi_{, j}(x) \equiv 0$ ed esista un indice $l$ per cui non risulti $\varphi_{l, j}(x) \equiv 0$ ed essendo $b$ un punto di continuità di $\varphi_{i l}(x)$, si abbia $\varphi_{i l}(b)>0$. Dico che sarà ancora $y_{i}(b)>0$. Ed invero, se fosse $y_{i}(b)=0$, dalle $(26)$ si dedurrebbe $\varphi_{i l}(b) y_{l}(b)=0$, e quindi $y_{l}(b) \neq 0$, donde

$$
\varphi_{l}(x) \equiv 0, \quad \varphi_{l h}(x) y_{h}(x) \equiv 0 \quad(h=1, \ldots, p),
$$

e quindi $\varphi_{l, j}(x) y_{j}(x) \equiv 0$, cioè l'assurdo $\varphi_{l, j}(x) \equiv 0$. Si ha dunque il teorema:

VIII. Nelle ipotesi ammesse per il sistema (23), per due certi indici i $e \mathrm{j}$ si abbia

$$
y_{j}(a)>0, \quad y_{i}(a)=0, \quad \varphi_{i}(x) \equiv 0,
$$

supposto b al finito, si avrà $\mathrm{y}_{\mathrm{i}}(\mathrm{b})>0$, quando o $\varphi_{\mathrm{ij}}(\mathrm{x})$ non è identicamente nulla in (a, b), oppure quando per un certo indice $1 \neq \mathrm{i}, \grave{e} \varphi_{1 j}(\mathbf{x})$ non identicamente nulla, ed essendo b un punto di continuità di $\varphi_{\mathrm{il}}(\mathrm{x})$ si $h a \varphi_{\mathrm{il}}(\mathrm{b})>0$.

I teoremi VII e VIII hanno notevoli corollari concernenti problemi di integrazione relativi ai sistemi lineari:

$$
y_{h}{ }^{\prime}=\gamma_{h}(x)+\sum_{k=1}^{p} \varphi_{h k}(x) y_{k}, \quad(h=1, \ldots, p)
$$

essendo $\gamma_{h}(x)$ e $\varphi_{h k}(x)$ assegnate funzioni generalmente continue in $(a, b)$ ed 
ivi sommabili, con prescritte ulteriori condizioni lineari per le funzioni $y_{h}(x)$, supposto, per semplificare, anche $b$ al finito, e vogliamo dedicare il presente paragrafo a qualcuno di tali corollari, esaminando varii tipi di dette condizioni.

Si ha subito, anzitutto, il teorema:

IX. Se si ha, in (a, b),

$$
p_{h k}(x) \geq 0, \quad(h \neq k ; h, k=1, \ldots, p),
$$

ad ogni soluzione $\left[\mathrm{y}_{\mathrm{h}}(\mathrm{x})\right]$ del sistema (27) si possono prescrivere ad arbitrio $i$ valori in a di $\mathrm{p}-1$ delle funzioni $\mathrm{y}_{\mathrm{h}}$ e della rimanente in $\mathrm{b}$ (in seguito a che la soluzione riesce determinata).

Si prescrivano, invero, le condizioni terminali (lineari)

e $\operatorname{siano}$

$$
y_{1}(a)=\alpha_{1}, y_{2}(a)=\alpha_{2}, \ldots, y_{p-1}(a)=\alpha_{p-1}, y_{p}(b)=\beta,
$$

$$
\left[u_{1 h}\right],\left[u_{2 h}\right], \ldots,\left[u_{p h}\right], \quad(h=1, \ldots, p),
$$

$p$ soluzioni fondamentali per il sistema (27), ridotto omogeneo, avendosi

$$
u_{i h}(a)\left\{\begin{array}{ll}
=1, & \text { se } i=h, \\
=0, & \text { se } i \neq h,
\end{array} \quad(i, h=1, \ldots, p),\right.
$$

e $\left[Y_{h}\right]$ la soluzione del sistema (27) verificante le condizioni iniziali

$$
Y_{h}(a)=0, \quad(h=1, \ldots, p) .
$$

La soluzione del sistema (27), determinata dalle condizioni iniziali

è data da

$$
y_{1}(a)=\alpha_{1}, y_{2}(a)=\alpha_{2}, \ldots, y_{p-1}=\alpha_{p-1}, y_{p}(a)=\lambda \text {, }
$$

$$
\left\{\begin{array}{l}
y_{1}(x)=Y_{1}(x)+\sum_{h=1}^{p-1} \alpha_{k} u_{h_{1}}(x)+\lambda u_{p_{1}}(x) \\
\cdot \cdot \cdot \cdot \cdot \cdot \cdot \cdot \\
y_{p}(x)=Y_{p}(x)+\sum_{h=1}^{p-1} \alpha_{h} u_{h p}(x)+\lambda u_{p p}(x)
\end{array}\right.
$$

e occorre e basta dare a $\lambda$ tale valore che si abbia $y_{p}(b)=\beta$, ciò che è evidentemente possibile, ed in un sol modo, poichè, in base al teor. VII, essendo $u_{p p}(a)=1, u_{p h}(a)=0(h \leqq p-1)$, riesce certamente $u_{p p}(b)>0$. Col cambiamento di variabile $x=a+b-\xi$, il teorema dimostrato fornisce il seguente:

IX'. Se si ha, in (a, b),

$$
\varphi_{h k}(x) \leqq 0, \quad(h \neq k ; h, k=1, \ldots, p),
$$

ad ogni soluzione $\left[\mathrm{y}_{\mathrm{h}}(\mathrm{x})\right]$ del sistema (27) si possono prescrivere ad arbitrio $i$ valori in $\mathrm{b} d i \mathrm{p}-1$ delle funzioni $\mathrm{y}_{\mathrm{h}}$ e della rimanente in $\mathrm{a}$. 
Per l'integrazione di un' unica equazione differenziale d'ordine $p$,

$$
y^{(p)}=\gamma(x)+\varphi_{0}(x) y+\varphi_{1}(x) y^{\prime}+\ldots+\varphi_{p-1}(x) y^{(p-1)}
$$

se ne deduce $\left({ }^{12}\right)$ :

$\mathrm{X}$. Se termine noto e coefficienti dell' equazione (29) sono generalmente continui e sommabili in (a, b), e si ha ivi

$$
\varphi_{k}(x) \geqq 0\left[(-1)^{p-k} \varphi_{k}(x) \geqq 0\right], \quad(k=0,1, \ldots, p-2),
$$

ad ogni soluzione $\mathrm{y}(\mathrm{x})$ dell' equazione si possono prescrivere ad arbitrio $i$ valor $i$ in a $[$ in $\mathrm{b}] d i \mathrm{p}-1$ delle funzioni $\mathrm{y}, \mathrm{y}^{\prime} \ldots, \mathrm{y}^{(\mathrm{p}-1)} e$ della rimanente in $\mathrm{b}$ $[$ in a $]\left({ }^{13}\right)$.

(12) Supposto $\varphi, \varphi_{0}, \ldots, \varphi_{p-2}, \varphi_{p-1}$ gencralmente continue in $(a, b)$ ed ivi sommabili e $\varphi, \varphi_{0}, \ldots, \varphi_{, r-2}$ non negative la soluzione $y(x)$ doll' equazione

$$
y^{(p)}=\varphi+\varphi_{0} y+\varphi_{1} y+\ldots+\varphi_{z}-2 y^{(p-2)}+\varphi_{p-1} y^{(p-1)}
$$

verifieante le condizioni iniziali

verifica, in $(a, b)$ le limitazioni:

$$
y^{(k)}(a)=x_{k} \geqq 0 \quad(h=0,1, \ldots, p-1) .
$$

$$
\begin{aligned}
& y^{(p-1)}(x) \geqq\left(\alpha_{p-1}+\int_{a}^{x} p(s) e^{-\int_{a}^{s} \varphi_{p-1}(t) d t} d s\right) e^{\int_{p}^{x} \varphi_{p-1}(t) d t}, \\
& y^{(k)}(x) \geqq x_{k}+x_{k+1}(x-a)+\ldots+\alpha_{p-2} \frac{(x-\alpha) p-2-k}{(p-2-k) !}+ \\
& +\int_{a}^{x} \frac{(x-\xi)}{(p-2-2-k) !}\left(x_{p-1}+\int_{a}^{\xi} \varphi(s) e^{-\int_{a}^{\xi} \varphi_{p-1}(t) d t} d s\right) e^{\int_{a}^{\xi} \varphi_{p-1}(t) d t} d \xi \\
& (h=0,1, \ldots, p-2) \text {. }
\end{aligned}
$$

(13) Per l'equazione del second' ordine

$$
y^{\prime \prime}=\gamma+\varphi_{0} y+\varphi_{1} y^{\prime}
$$

si ha dunque che se $\varphi_{0}(x) \geqq 0$, nell'intervallo $(a, b)$, ad una sua soluzione si possono preserivere ad arbitrio, in uno degli estremi dell'intervallo, il valore di essa e nell'altro quello della derivata prima. Tale circostanza è da reputarsi nota, e, posto

si ricava dall'identitat

$$
\eta(x)=e^{-\int_{a}^{x} \varphi_{1}(\xi) d \xi},
$$

$$
\int_{a}^{b} \theta u^{\prime 2} d x+\int_{a}^{b} \theta \varphi_{0} u^{2} d x=0
$$

alla quale soddisfa una soluzione dell' equazione, ridotta omogenea, ehe sia nulla in uno degli estremi dell'intervallo e abbia nell' altro nulla la derivata prima. 
Il teorema VIII permette, in modo del pari immediato, di dimostrare anche il seguente:

$\mathrm{XI}$. Se per $\mathrm{h} \neq \mathrm{k}$, si ha $\varphi_{\mathrm{hk}}(\mathrm{x}) \geqq 0\left[\varphi_{\mathrm{hk}}(\mathrm{x}) \leqq 0\right]$ e per due certi indici $\mathrm{i}$ e $\mathrm{j}$ $(\mathrm{i} \neq \mathrm{j})$ il coefficiente $\varphi_{\mathrm{ij}}(\mathrm{x})$ non è identicamente nullo, oppure, per un certo in. dice $1 \neq \mathrm{i}$ è $\rho_{1 j}(\mathrm{x})$ non identicamente nullo, ed essendo $\mathrm{b}$ [ed essendo a] un punto dt continuitò di $\varphi_{\mathrm{il}}(\mathrm{x})$, si ha $\psi_{\mathrm{i} 1}(\mathrm{~b})>0\left[\varphi_{\mathrm{il}}(\mathrm{a})<0\right]$, ad ogni soluzione $\left[\mathrm{y}_{\mathrm{h}}\right]$ del sistema (27) si possano assegnare ad arbitrio $i$ valori in a $e$ in b della funzione $\mathrm{y}_{\mathrm{i}} e$ i valori in a $[$ in $\mathrm{b}]$ delle funzioni $\mathrm{y}_{1}, \mathrm{y}_{\mathrm{z}}, \ldots, \mathrm{y}_{\mathrm{p}}$, privața della $\mathrm{y}_{\mathrm{j}}$.

Sia, per esempio, $i=1, j=p$, e si assegnino le condizioni terminali

$$
\begin{gathered}
y_{1}(a)=\alpha, \quad y_{1}(b)=\beta, \\
y_{2}(a)=\alpha_{2}, y_{3}(a)=\alpha_{3}, \ldots, y_{p-1}(a)=\alpha_{p-1} ;
\end{gathered}
$$

la soluzione $\left[y_{h}(x)\right]$ del sistema (27), le cui componenti sono date dalle (28), verifica tutte le condizioni in $a$, ed essa pertanto verificherà le prescritte condizioni terminali se (e sola se) si puo determinare il parametro $\lambda$ in guisa che si abbia

$$
Y_{1}(b)+\sum_{k=1}^{p-1} x_{k} u_{k_{1}}(b)+\lambda u_{p_{1}}(b)=\beta_{1}
$$

cio che è evidentemente possibile, od in un sol modo, poichè in base al teor. VIII, essendo $u_{, p}(a)=1, u_{r, h}(a)=0(h \leqq p-1)$, riesce, nelle ipotesi ammesso, $u_{p_{1}}(b)>0$.

Un'interpretazione immediata del teorema XI per l'equazione differen. ziale (29) non darebbe completezza di risultati e conviene, a questo scopo, esaminare direttamente su tale equazione il problema della determinazione di una sua soluzione alla quale si preserivano in $a e$ in $b$ i valori di una delle funzioni $y, y^{\prime}, \ldots, y^{(h-1)}$ e, nell' ipotesi $\varphi_{h}(x) \geq 0$ in $(a, b)(h=0,1, \ldots, p-2)$ i valori in $a$ delle rimanenti funzioni private di una. Dati due indici $i$ e $j$, scelto fra $0,1, \ldots, p-1$, diversi fra loro, si voglia costruire una soluzione della (29) soddisfacente le condizioni terminali

$$
\begin{aligned}
& y^{(i)}(a)=\alpha_{i}, \quad y^{(i)}(b)=\beta, \\
& y^{(k)}(a)=\alpha_{k}, \quad \text { per } k \neq j .
\end{aligned}
$$

Introdotto il sistema fondamentale $u_{1}, u_{2}, \ldots, u_{p}$, di soluzioni della (29) ridotta omogenea, verificanti le condizioni iniziali

$$
u_{h}^{(h)}(a)\left\{\begin{array}{l}
=0, \\
=1,
\end{array} \text { se } k \neq h-1, \quad(h=1, \ldots, p, k=0,1, \ldots, p-1),\right.
$$

e la soluzione $Y(x)$ della $(29)$, per la quale

$$
Y^{(k)}(a)=0, \quad(h=0,1, \ldots, p-1),
$$


ponendo

$$
y=Y(x)+\sum_{\substack{k=0 \\ k \neq j}}^{p-1} \alpha_{k} u_{1+k}(x)+\lambda u_{1+j}(x)
$$

riescono, da tale soluzione della (29), comunque si assuma il parametro $\lambda$, soddisfatte le prescritte condizioni in $a$ e volendo soddisfare quella in $b$ occorre e basta di determinare $\lambda$ in maniera che si abbia

$$
Y^{(i)}(b)+\sum_{\substack{k=0 \\ k \neq j}}^{p-1} \alpha_{k} u_{1+k}^{(i)}(b)+\lambda u_{1+j}^{(i)}(b)=\beta,
$$

ciò che si potrà certo fare se risulta $u_{1+j}^{(i)}(b) \neq 0$. Sappiamo che $u_{1+j}^{(i)}(b) \geq 0 \mathrm{e}$ che il segno $=$ può sussistere nel solo caso che si abbia $u_{1+j}^{(i)}(x) \equiv 0$ in $(a, b)$. Se $i>j$ da tale identità segue che $u_{1+j}^{(j)}(x) \equiv 0$, in contraddizione con la $u_{1+j}^{(j)}(a)=1$. Se $i>j$ dalla detta identità segue ché $u_{1+j}(x)$ è un polinomio di grado $i-1$, al più, e si ha quindi, date le condizioni iniziali,

e pertanto

$$
u_{t+j}(x)=\frac{(x-a)^{j}}{j !}
$$

$$
\varphi_{n}(x) \frac{(x-a)^{j}}{j !}+\varphi_{1}(x) \frac{(x-a)^{j-1}}{(j-1) !}+\cdots+\varphi_{j}(x)=0
$$

donde, per essere $j \leqq p-2$,

$$
\varphi_{1}(x) \equiv \varphi_{1}(x) \equiv \ldots \equiv \varphi_{j}(x) \equiv 0 .
$$

Abbiamo dunque il notevole teorema:

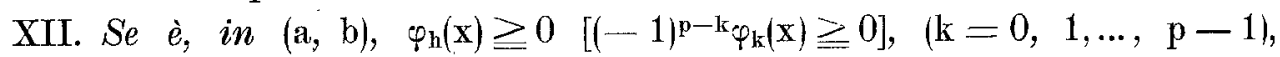
fissati due indici diversi $\mathrm{i}$ e $\mathrm{j}$, scelti fra $0,1, \ldots, \mathrm{p}-1$, ad una soluzione della (29) si possano prescrivere ad arbitrio $i$ valori in a $e$ in $\mathrm{b} d i \mathrm{y}^{(\mathrm{i})}$ e in a $[i n \mathrm{~b}]$ quelli delle funzioni $\mathrm{y}, \mathrm{y}^{\prime}, \ldots, \mathrm{y}^{(\mathrm{p}-1)}$, privata della $\mathrm{y}^{(\mathrm{j})}$, se $\mathrm{i}<\mathrm{j}$, e, nell'altro caso, se non tutti $i$ coefficienti $\varphi_{0}, \varphi_{1}, \ldots, \varphi_{j}$ sono identicamente nulli in $(\mathrm{a}, \mathrm{b})\left({ }^{1+}\right)$.

(14) Per $i>j$ e per $\varphi_{0} \equiv \varphi_{1} \equiv \ldots \equiv \varphi_{j} \equiv 0$, il problema considerato possiede d'altronde l'autosoluzione $(x-a)^{j}$ e i dati sono legati dalla relazione

$$
Y(b)+\sum_{\substack{h=0 \\ k \neq j}}^{p-1} \alpha_{h} u_{1+h}^{(i)}(b)=\beta
$$

Per l'equazione differenziale del $2^{\circ}$ ordine

$$
y^{\prime \prime}=\gamma+\varphi_{0} y+\varphi_{1} y^{\prime}
$$

con $\varphi_{0} \geqq 0$, si possono dunque, ad una. sua soluzione, prescriverne ad arbitrio in $a$ e in $b$ 
I risultati ottenuti forniscono teoremi d'esistenza solo in problemi per il sistema (27), con condizioni (lineari) terminali dalle quali in uno degli estremi dell' intervallo $(a, b)$ viene prescritto il valore di una sola delle funzioni incognite, ma i teoremi VII e VIII possono condurre anche a teoremi d'esistenza nel caso che dalle dette condizioni vengano preseritti in entrambi gli estremi i valori a più di una delle funzioni incognite. Ed eceo come. Sia $p=r+s$, $r \geqq 2, s \geqq 2$, e le condizioni preserivano foio che si può sempre supporre senza ledere le generalita) in $a$ i valuri delle funzioni

$$
y_{1}, y_{2}, \ldots, y_{r},
$$

$e$, detto $i_{1} i_{2} \ldots i_{s}$ una fissata combinazione di classe $s$, priva di inversione, degli indici $1,2, \ldots, p\left({ }^{15}\right)$, in $b$ i valori delle funzioni

e si abbia

$$
y_{i_{1}}, y_{i_{2}}, \ldots, y_{i_{\varepsilon}}
$$

$$
\begin{aligned}
& y_{1}(a)=\alpha_{1}, y_{2}(a)=\alpha_{2}, \ldots, y_{r}(a)=\alpha_{r}, \\
& y_{i_{1}}(b)=\beta_{1}, y_{i_{2}}(b)=\beta_{2}, \ldots, y_{i_{s}}(b)=\beta_{s},
\end{aligned}
$$

Introdotto il solito sistema fondamentale $\left[u_{1 h}\right],\left[u_{2 h}\right], \ldots,\left[u_{p_{h}}\right]$ di soluzioni del sistema (27), ridotto omogeneo, e la soluzione $\left[Y_{h}\right]$ del sistema (27), posto

$$
y_{h}=Y_{h}(x)+\sum_{m=1}^{r} \alpha_{m} u_{m h}(x)+\sum_{n=1}^{s} \lambda_{,} u_{r+n, h}(x), \quad(h=1, \ldots, p),
$$

da tale soluzione $\left[y_{h}\right]$ del sistema (27) verranno verificate, comunque si assumano i parametri $\lambda_{1}, \lambda_{2}, \ldots, \lambda_{s}$, le $(30)$ e si potranno verificare anche le (31) allora e allora soltanto che riesca possibile la determinazione dei detti parametri in maniera da soddisfare le $s$ equazioni lineari:

$$
\begin{aligned}
& \sum_{n=1}^{s} u_{r+n, i_{1}}(b) \lambda_{n}=\beta_{1}-\sum_{m=1}^{r} \alpha_{m} u_{m i_{1}}(b)-Y_{i_{1}}(b), \\
& \sum_{n=1}^{s} u_{r+n, i_{2}}(b) \lambda_{n}+\beta_{2}-\sum_{m=1}^{r} \alpha_{m} u_{m i_{2}}(b)-Y_{i_{2}}(b), \\
& \begin{array}{c}
\cdot \cdot \cdot \cdot \cdot \cdot \cdot \cdot \cdot \cdot \cdot \cdot \cdot \cdot \cdot \\
\sum_{n=1}^{s} u_{s+n, i_{s}}(b) \lambda_{,}=\beta_{s}-\sum_{m=1}^{r} \alpha_{m} u_{m i s}(b)-Y_{i_{s}}(b),
\end{array}
\end{aligned}
$$

i valori e. se $\varphi_{0}(x)$ non $\dot{\theta}$ identicamente nulla, quelli della derivata prima. Ciò è notissimo e lo fornisce anche l'identita ricordata nella nota $\left({ }^{13}\right)$. Per l'equazione del $3^{\circ}$ ordine

$$
y^{\prime \prime \prime}=\gamma+\varphi_{0} y+\varphi_{1} y^{\prime}+\varphi_{2} y^{\prime \prime}
$$

con $f_{0} \geqq 0, f_{1}>0\left[\varphi_{0} \leqq 0, \varphi_{1}>0\right]$, si possono, ad una sua soluzione, preserivere ad arbitrio $\mathrm{i}$ valori in $a \mathrm{e}$ in $b e$ in $a$ [in $b]$ di $y^{\prime} \circ$ di $y^{\prime \prime}$, oppure $\mathrm{i}$ valori in $a \in$ in $b$ di $y^{\prime} e$ in $a$ [in $b]$ di $y$; se non è $\varphi_{0} \equiv \varphi_{1}=0$, i valexi in $a$ e in $b$ di $y^{\prime \prime}$ e in $a$ [in $\left.b\right]$ di $y$; se non è $\varphi_{0} \equiv 0$, i valori in $a$ e in $b$ di $y^{\prime}$ o di $y^{\prime \prime}$ e in $a$ [in $\left.b\right]$, rispettiramente, di $y^{\prime \prime}$ o di $y^{\prime}$.

(15) Nel seguito quando parleremo di una combinazione di determinati indict, inten. deremo sempre che essa sia priva di inversioni. 
ciò che avverrà certo se il determinante

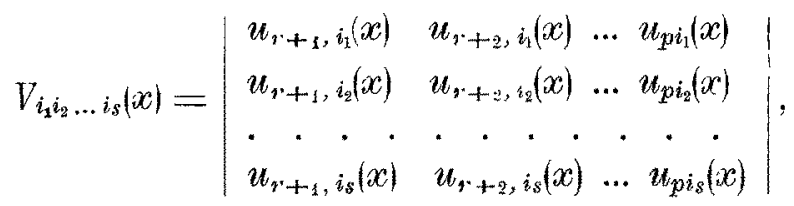

risulta in $b$ diverso da zero. Tale determinante è il minore della matrice di $p$ righe e di $s$ colonne

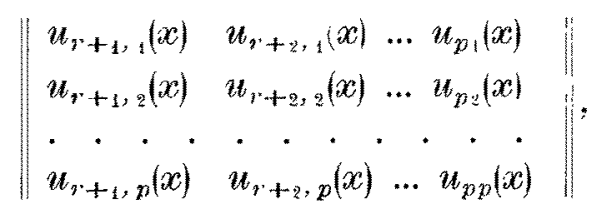

formato dalle stesse colonne e dalle righe $i_{1}^{m a}, i_{z}^{m a}, \ldots, i_{s}^{m a}$. Detta $\vee_{1} v_{2} \ldots v_{s}$ una variabile combinazione di classe $s$ degli indici $1,2, \ldots, p$, consideriamo i $\left(\begin{array}{l}p \\ s\end{array}\right)$ determinanti minori $V_{\nu_{1} \gamma_{s} \ldots \nu_{s}}(x)$ della matrice $(32)$ formati dalle $s$ colonne della matrice e dalle righe $v_{1}^{m a}, v_{2}^{m a}, \ldots, v_{s}^{m a}$. $\mathrm{E}$ subito visto che tali funzioni verificano un sistema di equazioni differenziali lineari omogenee del tipo:

$$
\frac{d}{d x} V_{v_{1} \nu_{2} \ldots \nu_{s}}=\left(\varphi_{v_{1} \nu_{1}}+\varphi_{\nu_{2} \nu_{2}}+\ldots+\varphi_{v_{s} \nu_{s}}\right) V_{v_{1} \nu_{2} \ldots v_{s}}+\sum_{v_{1}^{\prime} \nu_{z}^{\prime} \ldots v_{s}^{\prime}} \varepsilon_{h k} \varphi_{h k} V_{v_{1}^{\prime} v_{2}^{\prime} \ldots v_{s}^{\prime}}
$$

ove gli indici $h$ e $k$ sono determinate funzioni (di valore diverso)

$$
h=h\left(v_{1} v_{2} \ldots v_{s}, v_{1}^{\prime} v_{2}^{\prime} \ldots v_{s}^{\prime}\right), \quad k=k\left(v_{1} v_{2} \ldots v_{s}, v_{1}^{\prime} v_{2}^{\prime} \ldots v_{s}^{\prime}\right),
$$

dalle combinazioni distinte $v_{1} v_{z} \ldots v_{s}, v_{1}^{\prime} v_{2}^{\prime} \ldots v_{s}^{\prime}$, la sommatoria essendo estesa a tutte le combinazioni $\nu_{1}{ }^{\prime} v_{2}^{\prime} \ldots v_{s}{ }^{\prime}$ diverse dalla $\nu_{1} \nu_{2} \ldots v_{s}$, ed essendo $\varepsilon_{h k}$ una quantità che prende soltanto i valori - $1,0,1$. Poichè

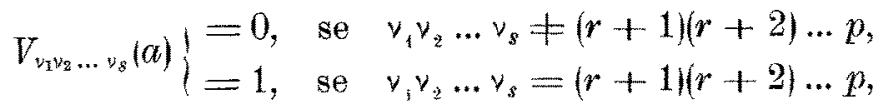

i teoremi VII e VIII consentono di enunciare il seguente:

XIII. Se, comunque si assumano le combinazioni $\nu_{1} v_{2} \ldots \nu_{s}, \nu_{1}^{\prime} \nu_{2}^{\prime} \ldots \nu_{s}^{\prime}$, degli indici $1,2, \ldots, \mathrm{p}$, si ha, in $(\mathrm{a}, \mathrm{b})$,

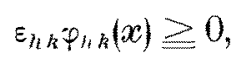

ad ogni soluzione del sistema (27) si possono prescrivere ad arbitrio in a $i$ 
valori di $\mathrm{r}$ fra le funzioni componenti e in b delle rimanenti $\mathrm{s}=\mathrm{p}-\mathrm{r}\left({ }^{(6)}\right)$. Nella stessa ipotesi, comunque si fissino due combinazioni $\dot{\mathrm{i}}_{\mathrm{t}} \mathrm{i}_{2} \ldots \mathrm{i}_{\mathrm{r}}, \mathrm{j}_{1} \mathrm{j}_{2} \ldots \mathrm{j}_{\mathrm{s}}$, aventi elementi comuni, detta $\mu_{1} \mu_{z} \ldots \mu_{\$}$ la complementare della prima, se il coefficiente

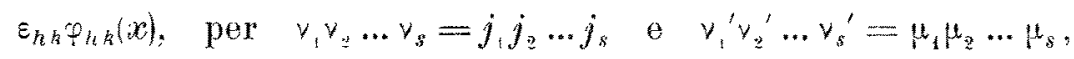

non è identicamente nullo, oppure, indicando con $1_{1} 1_{z} \ldots 1_{s}$ nna certa combinazione diversa dalla $\mathrm{j}_{1} \mathrm{j}_{2} \ldots \mathrm{j}_{\mathrm{s}}$, il coefficiente

$$
\varepsilon_{h k} \varphi_{h k}(x) \text {, per } \quad v_{1} \gamma_{z} \ldots v_{s}=l_{1} l_{2} \ldots l_{s} \text { e } \quad v_{1}^{\prime} \gamma_{2}^{\prime} \ldots, v_{s}^{\prime}=\mu_{1} k_{2} \ldots \mu_{s},
$$

non è identicamente nullo, essendo

$$
\varepsilon_{h k} \varphi_{h k}(b)>0 \text {, per } \quad v_{1} v_{2} \ldots v_{s}=j_{1} j_{2} \ldots j_{s} \text { e } \quad v_{1}^{\prime} \nu_{2}^{\prime} \ldots \nu_{s}^{\prime}=l_{1} l_{2} \ldots l_{s},
$$

supposto b un punto di continuità per tale funzione, ad ogni soluzione del sistema (27) si possono prescrivere ad arbitrio $i$ valori in a delle componenti $\mathrm{y}_{\mathrm{i}_{1}}, \mathrm{y}_{\mathrm{i}_{2}}, \ldots, \mathrm{y}_{\mathrm{i}_{\mathrm{r}}}$ e in $\mathrm{b}$ delle $\mathrm{y}_{\mathrm{j}_{1}}, \mathrm{y}_{\mathrm{j}_{2}}, \ldots, \mathrm{y}_{\mathrm{j}_{\mathrm{s}}}$.

Se, per esempio, si fa applicazione del teorema ora dato, nel caso $r=s=2$. si ha il seguente:

XIII'. Dato il sistema di quattro equazioni differenziali

$$
y_{h}^{\prime}=\gamma_{h}+\varphi_{k 4} y_{1}+\varphi_{k 2} y_{2}+\varphi_{k 3} y_{3}+\varphi_{k 4} y_{4}, \quad(h=1, \ldots, 4),
$$

nelle quattro funzioni incognite $\mathrm{y}_{1}, \mathrm{y}_{2}, \mathrm{y}_{3}, \mathrm{y}_{4}$, se. per una certa permutazione $\mathrm{i}_{1} \mathrm{i}_{2} \mathrm{i}_{3} \mathrm{i}_{4}$ degli indici $1,2,3,4$ si ha, in $(\mathrm{a}, \mathrm{b})$,

$$
\varphi_{i_{1} i_{2}}(x) \equiv \varphi_{i_{2} i_{1}}(x) \equiv \varphi_{i_{3} i_{4}}(x) \equiv \varphi_{i_{4} i_{i}}(x) \equiv 0
$$

e dei rimanenti elementi, non appartenti alla diagonale principale, del deter. minante

$$
\left|\begin{array}{ccc}
\varphi_{11} & \cdots & \varphi_{14} \\
\cdots & \cdots & \cdots \\
\varphi_{41} & \cdots & \varphi_{44}
\end{array}\right|
$$

due coniugati risultano, in ( $\mathrm{a}, \mathrm{b})$, non positivi e $i$ rimanenti non negativi, ad ogni soluzione del sistema si possono prescrivere ad arbitrio $i$ valori in a $d i$ due qualsivogliano componenti e in b quelli delle rimanenti.

Dette, invero, $\left[u_{h}\right]$ e $\left[v_{k}\right]$ due soluzioni del sistema $\left(27_{4}\right)$, ridotto omogeneo,

(10) È ben ovvio, ma ̀̀ bene notare, che ciò è anche possibile se, con un'opportuno

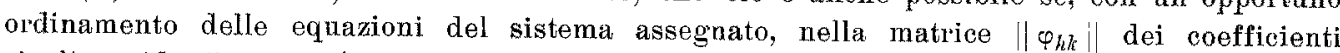
risultano identicamente nulli tutti gli elementi sitnati da una medesima parte di una delle diagonali. 
e posto, per ogni combinazione $v_{1} v_{2}$ degli indici $1,2,3,4$,

$$
V_{v_{1} \nu_{2}}=\left|\begin{array}{ll}
u_{\nu_{1}} & v_{v_{1}} \\
u_{\nu_{2}} & v_{v_{2}}
\end{array}\right| \text {, }
$$

si trova che le sei funzioni $V_{v_{1} y_{2}}$ verificano il seguente sistema omogeneo di equazioni differenziali :

$$
\left\{\begin{array}{l}
V_{12}^{\prime}=\left(\varphi_{14}+\varphi_{22}\right) V_{12}-\varphi_{13} V_{23}-\varphi_{14} V_{24}+\varphi_{23} V_{13}+\varphi_{24} V_{14}, \\
V_{13}{ }^{\prime}=\left(\varphi_{14}+\varphi_{33}\right) V_{13}+\varphi_{12} V_{23}-\varphi_{14} V_{34}+\varphi_{32} V_{12}+\varphi_{34} V_{14}, \\
V_{14}{ }^{\prime}=\left(\varphi_{11}+\varphi_{44}\right) V_{14}+\varphi_{12} V_{24}+\varphi_{13} V_{34}+\varphi_{12} V_{12}+\varphi_{43} V_{13}, \\
V_{23}^{\prime}=\left(\varphi_{22}+\varphi_{33}\right) V_{23}+\varphi_{21} V_{13}-\varphi_{24} V_{34}-\varphi_{31} V_{12}+\varphi_{34} V_{24}, \\
V_{24}^{\prime}=\left(\varphi_{22}+\varphi_{14}\right) V_{24}+\varphi_{21} V_{14}+\varphi_{23} V_{34}-\varphi_{14} V_{12}+\varphi_{43} V_{23}, \\
V_{34}^{\prime}=\left(\varphi_{32}+\varphi_{41}\right) V_{34}+\varphi_{34} V_{14}+\varphi_{32} V_{24}-\varphi_{14} V_{13}-\varphi_{12} V_{23},
\end{array}\right.
$$

Ora, nelle ipotesi del teorema, se, come sempre si può ottenere a mezzo di un eventuale cambiamento di notazioni, si ha

$$
\begin{gathered}
\varphi_{13} \equiv \varphi_{31} \equiv \varphi_{24} \equiv \varphi_{42} \equiv 0 \\
\quad \varphi_{14} \leqq 0, \quad \varphi_{14} \leqq 0, \\
\varphi_{12} \geqq 0, \quad \varphi_{24} \geqq 0, \quad \varphi_{23} \geqq 0, \quad \varphi_{32} \geqq 0, \quad \varphi_{34} \geqq 0, \quad \varphi_{43} \geqq 0,
\end{gathered}
$$

al sistema (33) si applica il teor. VII e pertanto, se si pone, ad esempio,

$$
\begin{array}{ll}
u_{3}(a)=1, & u_{1}(a)=u_{2}(a)=u_{4}(a)=0, \\
v_{4}(a)=1, & v_{1}(a)=v_{2}(a)=v_{3}(a)=0,
\end{array}
$$

risulta $V_{3 i}(x)>0$ in tutto $(a, b)$, e si può quindi, ad una soluzione del sistema $\left(27_{4}\right)$, prescrivere ad arbitrio $\mathrm{i}$ valori in $a$ di $y_{4}$ e $y_{2}$ e in $b$ di $y_{3}$ e $y_{4}$. Verificandosi le (34) il sistema (27, assume la forma particolare:

$$
\begin{cases}y_{1}^{\prime}=\gamma_{1}+\varphi_{11} y_{1}+\varphi_{12} y_{2}+\varphi_{14} y_{4}, & \text { (manca } \left.y_{3}\right) \\ y_{2}^{\prime}=\gamma_{2}+\varphi_{24} y_{1}+\varphi_{22} y_{2}+\varphi_{23} y_{3}, & \text { (manca } \left.y_{4}\right) \\ y_{3}^{\prime}=\gamma_{3}+\varphi_{32} y_{2}+\varphi_{33} y_{3}+\varphi_{34} y_{4}, & \text { (manca } \left.y_{1}\right) \\ y_{4}^{\prime}=\gamma_{4}+\varphi_{4} y_{1}+\varphi_{43} y_{3}+\varphi_{44} y_{4}, & \text { (manca } \left.y_{2}\right)\end{cases}
$$

e per un tale sistema usufruendo del teor. VIII applicato al sistema (33), vogliamo esaminare inoltre la possibilità di assegnare $\mathrm{i}$ valori in $a$ e in $b$ di una medesima delle funzioni $y_{h}$, cioè, se, ad esempio, si assegnano in $a$ i valori di $y_{1}$ e di $y_{2}$, la possibilità di dare in $b$ i valori di $y_{2}$ e $y_{3}$ o di $y_{2}$ e $y_{4}$ o di $y_{1}$ e $y_{3}$ o di $y_{1}$ e $y_{4}$ o, infine, di $y_{1}$ e $y_{2}$. Tale possibilità sussiste se, rispettivamente, riesce $V_{23}(b)>0 \quad$ o $V_{24}(b)$ o $V_{13}(b)>0 \quad$ o $\quad V_{14}(b)>0 \quad 0$, infine, $V_{12}(b)>0$. Pertanto, poichè $V_{34}(a)>0$, conformemente al teor. VIII 
applicato al sistema (33), nelle ipotesi che si verifichino le (34) e (35), si ha, per esempio, che:

$\mathrm{XIII}^{\prime}$. Se $\varphi_{* 3}$ non è identicamente nulla in (a, b), ad una soluzione del sistema (27,') si possono prescrivere ad arbitrio $i$ valori in a $e$ in $\mathrm{b} d i \mathrm{y}_{2}$, in a $d i \quad \mathrm{y}_{1}$ e in $\mathrm{b} d i \mathrm{y}_{4}$. Se $\mathrm{b}$ è punto di continuitò $d i \varphi_{21}$ ed é $\varphi_{21}(\mathrm{~b})>0$ $e \varphi_{14}$ non identicamente nulla, oppure $\mathrm{b}$ è punto di continuità di $\varphi_{34}$ ed è $\varphi_{34}(b)>0$ e $\varphi_{z 3}$ non identicamente nulla, si possono prescrivere ad arbitrio $i$ valori in a $e$ in $\mathrm{b} d \dot{\mathrm{y}} \mathrm{y}_{2}$, in a $d i \mathrm{y}_{1}$ e in $\mathrm{b} d i \mathrm{y}_{3}$. Se $\mathrm{b}$ è punto di continuità $d i \varphi_{14}$ ed $\grave{e} \varphi_{14}(b)<0$ e $\varphi_{23}$ non identicamente nulla, oppure $\mathrm{b}$ è punto di continuità di $\varphi_{23}$ ed $\grave{e} \varphi_{2:}(b)>0$ e $\varphi_{14}$ non identicamente nulla, si possono prescrivere ad arbitrio $i$ valori in a $e$ in $\mathrm{b} d i \mathrm{y}_{1}$ e di $\mathrm{y}_{2}$ ( $\left.{ }^{17}\right)$.

Con la solita posizione $y_{1+k}=y^{(k)}(k=0,1,2,3)$, l' equazione differenziale del quart' ordine

$$
y^{1 \nabla}=\gamma+\varphi_{0} y+\varphi_{1} y^{\prime}+\varphi_{2} y^{\prime \prime}+\varphi_{3} y^{\prime \prime \prime}
$$

si traduce, quando sia $\varphi_{t} \equiv 0$, nel sistema, del tipo $\left(27_{4}^{\prime}\right)$,

$$
\left\{\begin{array}{l}
y_{1}^{\prime}=y_{2}, \\
y_{2}^{\prime}=y_{3}, \\
y_{3}^{\prime}=y_{4}, \\
y_{4}{ }^{\prime}=\gamma+\varphi_{0} y_{1}+\varphi_{2} y_{3}+\varphi_{3} y_{4},
\end{array}\right.
$$

che verificherà le ipotesi del teor. XIII' quando sia, in $(a, b) . \varphi_{0} \leqq 0$ e $\varphi_{2} \geqq 0$, onde segue che ad una soluzione dell' equazione del $4^{\circ}$ ordine

$$
y^{\mathrm{Iv}}=\gamma(x)+\varphi_{0}(x) y+\varphi_{2}(x) y^{\prime \prime}+\varphi_{3}(x) y^{\prime \prime \prime},
$$

nell' ipotesi che sia in $(a, b)$

$$
\varphi_{0}(x) \leqq 0, \quad \varphi_{2}(x) \geqq 0,
$$

si possono prescrivere ad arbitrio, in $a$, i valori di due delle funzioni $y, y^{\prime}$, $y^{\prime \prime}, y^{\prime \prime \prime} \mathrm{e}$, in $b$, i valori delle rimanenti.

Al sistema $\left(27_{4}^{\prime \prime}\right)$, nell'ipotesi che valgano le (36), si applicano anche le due prime proposizioni del teor. XIII" e si ha dunque che, in tale ipotesi, ad una soluzione della $\left(29_{4}\right)$ si possono prescrivere ad arbitrio in $a$ e in $b \mathrm{i}$ valori di $y^{\prime}$, in $a$ di $y$ e in $b$ di $y^{\prime \prime \prime}$ oppure di $y^{\prime \prime}$. Non è però applicabile la terza proposizione di detto teorema e non si può dunque decidere per tale

(17) Se le $\varphi_{14}$ e $\varphi_{23}$ fossero identicamente nulle, le prime due equazioni del sistema $\left(27_{4}^{\prime}\right)$ verrebbero a legare fra loro esclusivamente $y_{1}$ e $y_{2}$ e pertanto i soli valori in $a$ (o in $b)$ di $y_{1} e$ di $y_{z}$ basterebbero a determinare tali funzioni. 
via della possibilità di preserivere ad una soluzione della $\left(29_{4}\right)$ i valori in $a$ e in $b$ di $y$ e di $y$ '. Per esaminare tale circostanza e tutte quelle che possono presentarsi nel tipo di questioni che stiamo trattando per l'equazione (29), occorre disimpegnarci dalle notazioni testè adottate ed osservare che per dne soluzioni $u$ e $v$ dell' equazione ridotta omogenea, posto

$$
u_{1+k}=a^{(k)}, \quad v_{1+k}=v^{(k)}, \quad(k=0,1,2,3),
$$

e definiti, come sopra, i determinanti $V_{v_{1} v_{2}}$, tali funzioni verificano il sistema

$$
\left\{\begin{array}{l}
V_{12}{ }^{\prime}=V_{13}, \\
V_{13}{ }^{\prime}=V_{23}+V_{14}, \\
V_{14}{ }^{\prime}=\varphi_{3} V_{14}+V_{24}+\varphi_{2} V_{13}, \\
V_{23}{ }^{\prime}=V_{24}, \\
V_{24}{ }^{\prime}=\varphi_{3} V_{24}+V_{34}-\varphi_{0} V_{12}+\varphi_{2} V_{23}, \\
V_{34}{ }^{\prime}=\varphi_{3} V_{34}-\varphi_{0} V_{12},
\end{array}\right.
$$

che si ricava da (33) ponendovi $\varphi_{12} \equiv \varphi_{23} \equiv \varphi_{34} \equiv 1, \quad \varphi_{44}=\varphi_{0}, \varphi_{43}=\varphi_{2}$, $\varphi_{44}=\varphi_{3}$, e identicamente nulli tutti gli altri coefficienti. Se sono soddisfatte le (36) a tale sistema si applica il primitivo teorema VII al quale ci basterà fare rioorso. Detto $h_{1} h_{2}$ e $k_{1} k_{2}$ due combinazioni degli indici $0,1,2,3$, detta $i_{1} i_{2}$ la combinazione complementare di $h_{1} h_{2}$, volendo decidere se, ad una soluzione della $\left(29{ }_{4}\right)$, si possono preserivere in $a$ i valori di

e in $b$ quelli di

$$
y^{\left(h_{1}\right)} \text { e } y^{\left(h_{2}\right)}
$$

$$
y^{\left(k_{1}\right)} \quad \text { e } y^{\left(k_{2}\right)}
$$

basterà vedere se, assunta per $u$ quella soluzione della $\left(29_{4}\right)$ ridotta omogenea che ha in $a$ la derivata $i_{1}{ }^{m a}$ di valore uno e tutte le altre nulle e per $v$ quella soluzione che ha in a la derivata $i_{2}{ }^{m a}$ di valore uno e tutte le altre nulle, riesce

Ora, si ha

$$
V_{\left(1+k_{3}\right)\left(1+k_{2}\right)}(b)>0
$$

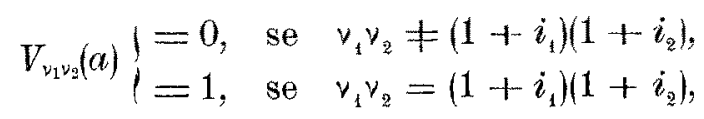

e pertanto, in base al teor. VII, si può affermare che, in $(a, b)$, riescirà $V_{v_{1} v_{2}}(x) \geqq 0$, per ogni combinazione $v_{1} v_{2}$ e quindi anche $V_{\left(1+k_{1}\right)\left(1+k_{2}\right)}(b) \geqq 0$, e $V_{\left(1+k_{1}\right)\left(k_{1}+k_{2}\right)}(b)=0$, solo quando sia $V_{\left(1+k_{2}\right)\left(1+k_{3}\right)}(x) \equiv 0$, e $V_{\left(1+i_{1}\right)\left(1+i_{2}\right)}(x)>0$. Da ciò, data la particolare forma del sistema $\left(33^{\prime}\right)$ è possibile, nelle sole 
ipotesi (36), o dedurre che si verifica la (37), o dare eriterii sufficienti perchè tale diseguaglianza sussista.

Si voglia, per esempio, decidere se si possono prescrivere in $a$ e in $b$ i valori di $y$ e di $y^{\prime}$. Si ba $h_{1}=k_{1}=0, h_{2}=k_{2}=1, i_{1}=2, i_{2}=3, \nabla_{34}(x)>0$,

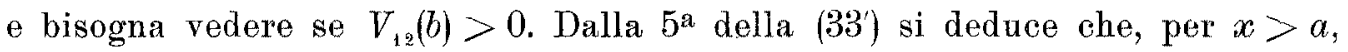
$V_{24}(x)>0$, dalla $4^{a}$ ohe $V_{23}(x)>0$, dalla $2^{a}$ che $V_{13}(x)>0$, e infine, dalla $1^{a}$ che $V_{12}(x)>0$, onde $V_{12}(b)>0$.

Si voglia decidere se si possono prescrivere in $a$ e in $b$ i valori di $y^{\prime \prime}$, in $a$ il valore di $y^{\prime}$ e in $b$ quello di $y^{\prime \prime}$. Si ha $h_{1}=1, h_{2}=2, k_{1}=2, h_{3}=3$, $i_{1}=0, i_{2}=3, V_{14}(x)>0$ e bisogna vedere se $V_{34}(b)>0$. Dalla $2^{a}$ delle $\left(33^{\prime}\right)$ si deduce che, per $x>a, V_{13}(x)>0$, dalla $1^{\mathrm{a}}$ che $V_{12}(x)>0$, e dall' ultima che $V_{34}(b)>0$ se $\varphi_{0}(x)$ non è identicamente nulla in $(a, b)$.

Si vogliano prescrivere in $a$ e in $b$ i valori di $y$ e di $y^{\prime \prime \prime}$. Si ha $h_{1}=k_{4}=0$, $h_{2}=k_{2}=3, i_{1}=1, i_{2}=2, V_{23}(x)>0$ e bisogna vedere se $V_{14}(b)>0$. Dalla $2^{a}$ delle $\left(33^{\prime}\right)$ si deduce che, per $x>a, V_{13}(x)>0$ e dalla $3^{\text {a }}$ che $V_{14}(b)>0$ 'se non è $\varphi_{2}(x) \equiv 0$. Sia $\varphi_{2}(x) \equiv 0$, dalla $1^{\text {a }}$ delle $\left(33^{\prime}\right)$ si deduce che, per $x>a$, $V_{12}(x)>0$, dalla $3^{a}$ che da $V_{14}(b)=0$, segue $V_{24}(x) \equiv 0 \quad \theta$ dalla $5^{\text {a }}$ che $\varphi_{\theta}(x) \equiv 0$. Si ha dunque $V_{14}(b)>0$, se non è $\varphi_{0}(x) \equiv \varphi_{:}(x) \equiv 0$, ece. .

L'esame di tutti i possibili casi conduce al seguente comprensivo notevole teorema :

XIV. Data l'equazione differenziale lineare del $4^{\circ}$ ordine $\left(29_{4}\right)$, nell' ipotesi che in $(\mathrm{a}, \mathrm{b})$ siano verificate le (36), ad una sua soluzione si possono prescri. vere ad arbitrio, in a, $i$ valori di una coppia di funzioni scelte fra le $y, y^{\prime}$, $\mathrm{y}^{\prime \prime}, \mathrm{y}^{\prime \prime \prime}$ e, in $\mathrm{b}, i$ valori della stessa o di un'altra coppia, nei seguenti casi:

quando non esiste un polinomio di secondo grado, non identicamente nullo, per il quale le stesse coppie assumano in a e in b valori nulli,

quando, esistendo un tale polinomio, e non ne esistendo uno di primo, non $\grave{e}$ in $(\mathrm{a}, \mathrm{b}), \quad \varphi_{\vartheta}(\mathrm{x}) \equiv \varphi_{2}(\mathrm{x}) \equiv 0 \quad$ (se $\varphi_{\mathrm{u}}(\mathrm{x}) \equiv \varphi_{2}(\mathrm{x}) \equiv 0$, il delto polinomio $\grave{e}$ autosoluzione del problema).

quando, esistendo un polinomio di grado non superiore al primo, non identicamente nullo, per il quale le dette coppie assumono in a $e$ in $\mathrm{b}$ valori

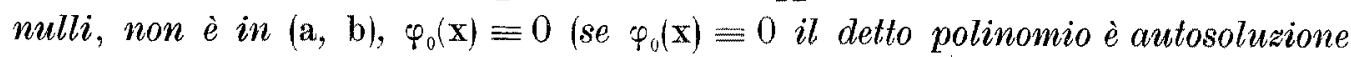
del problema).

Ritornando al sistema (27), i problemi da noi finora esaminati per la sua integrazione rientrano tutti come caso particolare nel seguente: Sono assegnati i sistemi di costanti

$$
\begin{aligned}
& a_{k 1}, a_{h 2}, \ldots, a_{k p}, \\
& b_{k 1}, b_{k z}, \ldots, b_{k p}, \\
& c_{h 1}, c_{h 2}, \ldots, ., c_{k p} \text {, }
\end{aligned}
$$

$(h=1,2, \ldots, r)$

$(h=1,2, . ., s)$,

$(h=1,2, \ldots, q)$, 
ed $\dot{e} r+s+2 q=p$, si suol costruire una soluzione $\left[y_{h}\right]$ del sistema che verifichi le $p$ condizioni lineari

$$
\begin{cases}\sum_{k=1}^{p} a_{k k} y_{k}(a)=\alpha_{k} & (h=1,2, \ldots, r), \\ \sum_{k=1}^{p} b_{k k} y_{k}(b)=\beta_{k} & (h=1,2, \ldots, s), \\ \sum_{k=1}^{p} c_{k k} y_{k}(a)=\alpha_{k}^{\prime}, \sum_{k=1}^{p} c_{h k} y_{k}(b)=\beta_{k}^{\prime}, & (h=1,2, \ldots, q) .\end{cases}
$$

Posto

$$
\begin{gathered}
t_{h k}=a_{h k}, \text { per } h=1,2, \ldots, r, \quad k=1,2 \ldots, p, \\
t_{r+h, k}=b_{h k}, \quad \text { per } h=1,2, \ldots, s, \quad k=1,2, \ldots, p, \\
t_{r+s+h, k}=c_{k k}, \quad \text { per } h=1,2, \ldots, q, \quad k=1,2, \ldots, p, \\
t_{r+s+q+h, k}\left\{\begin{array}{l}
\text { per } k=r+s+q+h, \quad h=1,2, \ldots, q, k=1,2, \ldots, p, \\
=1, \text { per } k=r+s+q+h,
\end{array}\right.
\end{gathered}
$$

si puó, senza ledere la generalita, supporre che il determinante

$$
\left|\begin{array}{ccc}
t_{1 \mathrm{t}} & \ldots & t_{1 p} \\
\ldots & \ldots & \ldots \\
t_{n 1} & \ldots & t_{n p}
\end{array}\right|
$$

sia diverso da zero, e allora effettuare nel problema il cambiamento delle funzioni incognite $y_{h}$ nelle $\eta_{h}$ a quelle legate dalle relazioni invertibili

$$
\left.\eta_{h}=\sum_{k=1}^{p} t_{k k} y_{k}, \quad \quad \mid h=1, \ldots, p\right)
$$

per il sistema

$$
\eta_{h}^{\prime}=\gamma_{h}^{*}+\sum_{k=1}^{p} \varphi_{h h_{k} \eta_{k}, \quad}, \quad(h=1, \ldots, p)
$$

che ne risulta, si ha il problema, già considerato, di costruirne una soluzione verificante le condizioni

$$
\begin{array}{lll}
\eta_{k}(a)=\alpha_{k}, & (h=1, \ldots, r), & \eta_{r+h}(b)=\beta_{h}, \quad(h=1,, \ldots, s), \\
& \eta_{r+s+h}(\alpha)=\alpha_{h}^{\prime}, & \eta_{r+s+h}(b)=\beta_{h}^{\prime},
\end{array}
$$

Cosi, per esempio, per l'integrazione dell' equazione del second'ordine

$$
y^{\prime \prime}=\gamma+\varphi_{n} y+\varphi_{1} y^{\prime}
$$

con le condizioni

$$
\begin{aligned}
& a_{1} y(\alpha)+a_{2} y^{\prime}(a)=\alpha, \\
& b_{1} y(b)+b_{2} y^{\prime}(b)=\beta,
\end{aligned}
$$

si trova che se $\mathrm{a}_{1} \mathrm{~b}_{2}-\mathrm{a}_{1} \mathrm{~b}_{2} \neq 0$, il problema ha sempre una (ed una sola) so- 
luzione quando sia, in (a, b),

$$
\begin{aligned}
& \left(a_{1} b_{2}-a_{2} b_{1}\right)\left(a_{2}^{2} \varphi_{11}(x)-a_{1} a_{2} \varphi_{1}(x)-a_{1}^{2}\right) \leqq 0, \\
& \left(a_{1} b_{2}-a_{2} b_{1}\right)\left(b_{2}^{2} \varphi_{1}(x)-b_{1} b_{2} \varphi_{1}(x)-b_{4}^{2}\right) \geqq 0 ;
\end{aligned}
$$

con le condizioni

$$
\begin{aligned}
& c_{1} y(a)+c_{2} y^{\prime}(a)=\alpha, \\
& c_{1} y(b)+c_{2} y^{\prime}(b)=\beta,
\end{aligned}
$$

si trova ehe, se $e_{1} \neq 0$, il problema ha sempre una (ed una sola) soluzione quando sia

$$
c_{1} \varphi_{1}(x) \geqq 0, \quad c_{4}\left(c_{z}^{*} \varphi_{0}(x)-c_{1} c_{2} \varphi_{1}(x)-c_{1}^{2}\right) \leqq 0,
$$

e non entrambe tali funzioni identicamente nulle (se queste funzioni fossero entrambe identicamente nulle, il problema arrebbe l'autosoluzione $\left.e^{-\left(c_{1} / c_{2}\right) x}\right)$.

Non vi è neppure difficoltà a stabilire, in base alla presente teoria, sriterii sufficienti d'esistenza per le soluzioni di problemi d'integrazione, riguardanti il sistema (27), nei quali oltre a condizioni del tipo delle (37), comparissero condizioni del seguente tipo integrale

$$
\sum_{k=1}^{p}\left(A_{k h} y_{k}(a)+B_{h k} y_{k}(b)+\int_{a}^{b} \Psi_{k h}(x) y_{k}(x) d x\right)=\rho_{k}, \quad(h=1, \ldots, x)
$$

il cui numero $x$ sommato a quella $r+s+2 q$ delle condizioni (37), dia un risultatio eguale a $p$, supposte $A_{h k}$ e $B_{k k}$ costanti e $\psi_{k k}(x)$ funzioni generalmente continue e sommabili in $(a, b)$.

Aggregate invero alle $y_{h}$ le $x$ funzioni incognite

$$
\eta_{h}(x)=\sum_{k=1}^{p}\left(A_{k k} y_{k}(a)+B_{h k} y_{k}(x)+\int_{a}^{x} \psi_{k k}(\xi) y_{k}(\xi) d \xi\right),
$$

il problema equivale al seguente, di tipo già considerato: $l^{\prime}$ integrazione del sistema di $p+x$ equazioni, nelle $p+x$ incognite $y_{1}, \ldots, y_{p}, \eta_{1}, \ldots, \eta_{x}$,

$$
\begin{cases}y_{k}{ }^{\prime}=\gamma_{k}+\sum_{k=1}^{p} \varphi_{k k} y_{k}, & (h=1, \ldots, p), \\ \eta_{k}{ }^{\prime}=\sum_{k=1}^{p}\left(\psi_{h k}+\sum_{i=1}^{p} B_{h i} \varphi_{i \hbar}(x)\right) y_{k}, & (h=1, \ldots, x),\end{cases}
$$

con le $p+x$ condizioni che si ottengono aggiangendo alla $r+s+2 q$ del tipo (37) le $2 x$ seguenti

$$
\eta_{h}(a)-\sum_{k=1}^{p}\left(A_{h k}+B_{h k}\right) y_{k}(a)=0 . \quad \eta_{h}(b)=\rho_{h}, \quad(h=1, \ldots, x) .
$$


Così, per esempio, per l'integrazione dell' equazione $\left(29_{3}\right)$ con le condizioni integrali

$$
\int_{a}^{b} \Psi(x) y(x) d x=\rho, \quad \int_{a}^{b} \psi_{1}(x) y^{\prime}(x) d x=\rho_{1}
$$

si trova, utilizzando il teor. XIII", che il problema ha sempre una (ed una sola soluzione) quando sia, in (a, b), $\varphi_{\mathrm{o}}(\mathrm{x}) \geqq 0$ e $\psi(\mathrm{x}), \Psi_{1}(\mathrm{x})$ di comune segno costante, quando non sono nulle, e nessuna di esse identicamente nulla.

Non possiamo, neppure in questo scritto, rinunziare ad un'osservazione con la quale porremo fine al presente paragrafo. I criterii sufficienti d'esistenza, che, in applicazione immediata della teoria svolta, possono ottener̀si coi procedimenti indicati, impongono, come abbiamo visto negli esempi trattati, forti restrizioni ai coefficienti delle equazioni del sistema da integrare, laddove, fissati comunque questi coefficienti, e assegnato a taluni di essi un fattore, da assumersi come parametro, si sa bene che i problemi lineari del tipo considerato hanno sempre soluzioni, all' infuori, eventualmente, per una certa successione di valori del parametro (antovalori). Ma gli è che, nelle applicazioni anche le più semplici, non si è affatto in grado di decidere se un dato valore del parametro sia o no autovalore, onde l'utilità dei criterii stabiliti, i quali, per altro, possono, col procedimento che andiamo ad osservare, molto aumentare il loro campo d'azione, fino a comprendere, come io presumo, tutti i casi nei quali sia possibile conseguire, teoremi che valgano in generale.

Ricordiamo che, dato il problema di integrare il sistema (27) con le condizioni del tipo considerato, esso possiede soluzione, comunque si assegnino i termini noti, allora e allora soltanto che il sistema omogeneo

$$
y_{k}{ }^{\prime}=\sum_{k=1}^{p} \varphi_{h k} y_{k}, \quad \quad(h=1, \ldots, p)
$$

non possiede che la soluzione nulla verificante le stesse condizioni ridotte omogenee. Assunte, comunque, le $p^{2}$ funzioni $\omega_{h k}(x)(h, k=1, \ldots, p)$, assolutamente continue in $(a, b)$ ed ivi dotate di derivate prime generalmente continue, con determinante

$$
\begin{array}{lll}
\omega_{11} & \ldots & \omega_{1 p} \\
\ldots & . & \ldots \\
\omega_{p 1} & \ldots & \omega_{p p}
\end{array}
$$

sempre non nulle in $(a, b)$, operiamo il cambiamento delle funzioni inco- 
gnite $y_{h}$ nelle $\eta_{h}$, a quelle legate dalle relazioni

$$
y_{h}=\sum_{i=1}^{p} \omega_{h i} \eta_{i} \quad(h=1, \ldots, p) ;
$$

detto $\Omega_{k k}$ il complemento algebrico di $\omega_{k k}$ nel determinante (38). diviso per il determinante stesso, il sistema $(27$,$) si traduce nel seguente$

$$
\eta_{i}^{\prime}=\sum_{j=1}^{p}\left(\sum_{h . k}^{1 . p} \varphi_{h k} \Omega_{h i} \omega_{k j} \quad \sum_{h=1}^{p} Q_{h i} \omega_{h j}^{\prime}\right) \eta_{j}=\sum_{j=1}^{p} \varphi_{i j}^{*} \eta_{j}
$$

e le condizioni lineari omogenee imposte alle $y_{h}$ in altrettante imposte alle $\eta_{i}$, dello stesso tipo. Se è possibile una scelta delle funzioni $\omega_{k k}$ in maniera the il sistema trasformato non abbia che la soluzione nulla verificante le condizioni trasformate, si potrà dire che il sistema primitivo non ha, pur esso, che la soluzione nulla verificante le condizioni primitive. E avremo perciò conseguito nuovi criterii sufficienti d'esistenza per le soluzioni di problemi relativi al sistema (27), se daremo criterii atti ad assicurare la possibilita di scegliere le funzioni $\omega_{k, k}(x)$ in maniera che il sistema omogeneo e le condizioni omogenee nelle $\eta$, presentino le particolarità imposte dai criterii sufficienti, di nullità della soluzione, derivanti dall'applicazione immediata della teoria svolta $\left({ }^{18}\right)$.

Si abbia, per esempio, $\lambda$ essendo un parametro, il sistema e le condizioni, in due funzioni incognito $y_{1}$ e $y_{2}$ :

$$
\left\{\begin{array}{l}
y_{1}^{\prime}=\varphi_{11} y_{1}+\lambda \varphi_{12} y_{2}, \\
y_{2}^{\prime}=\lambda_{21} y_{1}+\varphi_{22} y_{2}, \\
y_{1}(a)=y_{2}(b)=0 .
\end{array}\right.
$$

Si operi la trasformazione

$$
\begin{aligned}
& y_{1}=p \eta_{1}+u \eta_{2}, \\
& y_{2}=v \eta_{1}+q \eta_{2},
\end{aligned}
$$

con $p$ e $q$ costanti positivi e $u$ e $v$ funzioni della $x$, essendo

$$
\begin{gathered}
u(a)=v(b)=0, \\
p q-u(x) v(x)>0, \quad \text { in }(a, b) .
\end{gathered}
$$

(18) Indipendentemente da tale teoria si ha, efr. nota ${ }^{(15)}$, che l'unica soluzione del sistema (27 ) della quale le componenti $y_{1}, \ldots, y_{r}$ sono nulle in $a$ e le rimanenti in $b$, è la soluzione nulla, tutte le volte che si possono assumere le $\omega_{h k}(x)$ in maniera che nelia ma. trice $\left\|\varphi_{k h}\right\|$ dei coefficienti del sistema trasformato risultino identicamente nulli in $(a, b)$ tutti gli elementi situati da una medesima parte di una delle diagonali e si abbia

$$
\begin{array}{rrrr}
\omega_{h k}(a)=0, & \text { per } h \neq k & (h=1, . ., r ; k=1, \ldots, p), \\
\omega_{h k}(b)=0, & \text { per } h \neq k & (h=r+1, \ldots, p ; k=1, \ldots, p) .
\end{array}
$$


Si ha

$$
\begin{gathered}
\eta_{1}^{\prime}=\varphi_{11}^{*} \eta_{1}+\varphi_{12}^{*} \eta_{2}, \\
\eta_{2}^{\prime}=\varphi_{21}^{*} \eta_{1}+\varphi_{22}^{*} \eta_{2}, \\
\left.\left.\eta_{1}(a)=\eta_{2}\right) b\right)=0, \\
(p q-u v) \varphi_{12}^{*}=\left(\varphi_{11}-\varphi_{22}\right) q u+\lambda \varphi_{12} q^{2}-\lambda \varphi_{21} u^{2}-q u^{\prime}, \\
(p q-u v) \varphi_{21}^{*}=\left(\varphi_{z 2}-\varphi_{11}\right) p v+\lambda \varphi_{21} p^{2}-\lambda \varphi_{12} v^{2}-p v^{\prime},
\end{gathered}
$$

onde, in base ai teoremi IX e IX', che le (39) hanno l'unica soluzione nulla se è possibile determinare le costanti positive $p$ e $q$ e le funzioni $u(x)$ nulla in $a$ e $v(x)$, nulla in $b$, assolntamente continue in $(a, b)$ e con derivate prime generalmente continue, in modo che in $(a, b)$ si abbia

$$
\begin{gathered}
|u(x)|<p, \quad|v(x)|<q, \\
u^{\prime}=\lambda q\left(P+\varphi_{12}\right)+\left(\varphi_{11}-\varphi_{2,2}\right) u-\lambda \frac{\varphi_{21}}{q} u^{2}, \\
v^{\prime}=\lambda p\left(Q+\varphi_{21}\right)+\left(\varphi_{2,2}-\varphi_{11}\right) v-\lambda \frac{\varphi_{12}}{p} v^{2},
\end{gathered}
$$

con $P(x)$ e $Q(x)$ arbitrarie funzioni, di comune segno costante in $(a, b)$, quando non sono nulle, ivi generalmente continue e sommabili. Ora, data l'equazione differenziale di RICCATI

$$
u^{\prime}=\lambda \alpha+\beta u+\lambda \gamma u^{2}
$$

coi coefficienti $\alpha, \beta, \gamma$, sommabili in $(a, b)$, si trova $\left({ }^{19}\right)$ che essa possiede una soluzione $u$, assolutamente continua nell'intervallo $(\alpha, b)$, nulla in $a$, se il parametro $\lambda$ è così limitato

$$
|\lambda|<\frac{1}{2}\left(\int_{a}^{b}|\alpha| e^{-\int_{a}^{w} \beta d \xi} d x \cdot \int_{a}^{b}|\gamma| e^{\int_{a}^{x} \beta d \xi} d x\right)^{-\frac{1}{2}}
$$

valendo allora per la $u(x)$ la maggiorazione

$$
|u(x)| \leqq\left(\left.\frac{\int_{a}^{b}|\alpha| e^{-\int_{a}^{\infty} \beta d \xi} d x}{\int_{a}^{b} \gamma \mid e^{\int^{x} \beta d \xi} d x}\right|^{\frac{1}{2}} e^{\int^{a} \beta d \xi}\right.
$$

(19) Cfr. Proone, Nuova analisi esistenziale e quantitativa delle soluzioni dei sistemi di equazioni differenziali ordinarie $F$. Annali della R. Senola Normale Superiore di Pisa", vol. X (1941)]. 
e si perviene dunque al notevole risultato: Comunque si scelgano le funzioni $\mathrm{P}$ e $\mathrm{Q}$ di comune segno costante in (a, b), quando non sono nulle, le equazioni (39) hanno l' unica soluzione nulla se il parametro $\lambda$ è così limitato:

$$
|\lambda|\left\{\begin{array}{c}
<\frac{1}{2}\left(\int_{a}^{b}\left|P+\varphi_{12}\right| e_{a}^{\int_{a}^{x}\left(\varphi_{22}-\varphi_{11}\right) d \xi} d x \cdot \int_{a}^{b}\left|\varphi_{21}\right| e^{\int_{a}^{x}\left(\varphi_{11}-\varphi_{22}\right) d \xi} d x\right)^{-\frac{1}{2}}, \\
<\frac{1}{2}\left(\int_{a}^{b}\left|Q+\varphi_{21}\right| e^{\int_{a}^{x}\left(\varphi_{11}-\varphi_{22}\right) d \xi} d x \cdot \int_{x}^{b}\left|\varphi_{12}\right| e_{a}^{\int_{a}^{x}\left(\varphi_{22}-\varphi_{11}\right) d \xi} d x\right)^{-\frac{1}{2}},
\end{array}\right.
$$

e quindi in particolare, le equazioni

$$
\begin{aligned}
& y^{\prime \prime}=\lambda^{2} \varphi_{1} y+\varphi_{1} y^{\prime}, \\
& y(a)=y^{\prime}(b)=0,
\end{aligned}
$$

hanno l'unica soluzione nulla se il parametro $\lambda$ è così limitato:

$$
\lambda^{2}\left\{\begin{array}{c}
<\frac{1}{4}\left(\int_{a}^{b}|1+P| e^{\int_{a}^{\infty} \phi_{1} d \xi} d x \cdot \int_{a}^{b} \mid \varphi_{0} e^{-\int_{a}^{\infty} \varphi_{1} d \xi} d x\right)^{-1}, \\
<\frac{1}{4}\left(\int_{a}^{b}\left|\varphi_{0}+Q\right| e^{-\int_{a}^{\infty} d \varphi_{1} d} d x \cdot \int_{a}^{b \int_{a}^{\infty} e_{1} d \xi} d x\right)^{-1},
\end{array}\right.
$$

e pertanto, quando $\varphi_{0}(x) \leqq 0$ in $(\mathrm{a}, \mathrm{b})$, se

$$
\lambda^{2}<\frac{1}{4}\left(\int_{a}^{b} \int^{b \int_{0}^{\infty} \varphi_{1} d \xi} d x \cdot \int_{a}^{b}\left|\varphi_{0}\right| e^{-\int_{a}^{x} d x} d x\right)^{-t}
$$

Altro esempio, date le equazioni

$$
\left\{\begin{array}{l}
y^{\mathrm{TV}}=\varphi_{0} y+\varphi_{1} y^{\prime}+\varphi_{2} y^{\prime \prime}+\varphi_{3} y^{\prime \prime \prime}, \\
y(a)=y^{\prime}(a)=y^{\prime \prime}(a)=0, \quad y(b)=0
\end{array}\right.
$$

si ponga, con $\omega(x)>0$ in $(a, b)$,

si avranno per $\eta$ le equazioni

$$
y=\omega \eta
$$

$$
\begin{aligned}
& \eta^{\mathrm{IV}}=\varphi_{0}^{*} \eta+\varphi_{1}^{*} \eta^{\prime}+\varphi_{2}^{*} \eta^{\prime \prime}+\varphi_{3}^{*} \eta^{\prime \prime \prime}, \\
& \eta(a)=\eta^{\prime}(a)=\eta^{\prime \prime}(a)=0, \quad \eta(b)=0,
\end{aligned}
$$


con

$$
\begin{aligned}
& \omega \varphi_{0}^{*}=\varphi_{0} \omega+\varphi_{1} \omega^{\prime}+\varphi_{2} \omega^{\prime \prime}+\varphi_{3} \omega^{\prime \prime \prime}-\omega^{\mathrm{r}} \\
& \omega \varphi_{1}^{*}=\varphi_{1} \omega+2 \varphi_{2} \omega^{\prime}+3 \varphi_{3} \omega^{\prime \prime}-4 \omega^{\prime \prime \prime} \\
& \omega \varphi_{2}^{*}=\varphi_{2} \omega+3 \varphi_{2} \omega^{\prime}-6 \omega^{\prime \prime} \\
& \omega \varphi_{3}^{*}=\varphi_{3} \omega-4 \omega^{\prime}
\end{aligned}
$$

onde, in base al teor. XII si ha che: Le equazioni (40) possiedono l'umica soluzione nulla se esiste una funzione a positiva in (a, b), con derivata terza assolutamente continua e quarta generalmente contimua, per la quale, in (a, b), riesca

$$
\begin{array}{ll}
\varphi_{1} \omega+\varphi_{1} \omega^{\prime}+\varphi_{2} \omega^{\prime \prime}+\varphi_{3} \omega^{\prime \prime \prime}-\omega^{1 v} & \geqq 0, \\
\varphi_{1} \omega+2 \varphi_{2} \omega^{\prime}+3 \varphi_{3} \omega^{\prime \prime}-4 \omega^{\prime \prime \prime} & \geq 0, \\
\varphi_{2} \omega+3 \varphi_{3} \omega^{\prime}-6 \omega^{\prime \prime} & \geq 0,
\end{array}
$$

in particolare, posto $\omega=\mathrm{e}^{\alpha \mathrm{x}}$, se esiste una costante a per la quale riesca

$$
\begin{array}{ll}
\varphi_{1}+\alpha \varphi_{1}+\alpha^{2} \varphi_{2}+\alpha^{3} \varphi_{3}-\alpha^{4} \geq 0, \\
\varphi_{1}+2 \alpha \varphi_{2}+3 \alpha^{2} \varphi_{3}-4 \alpha^{3} & \geq 0, \\
\varphi_{3}+3 \alpha \varphi_{3}-6 \alpha^{2} & \geq 0 .
\end{array}
$$

Così, data ancora l'equazione differenziale (40) con le condizioni

$$
y(\alpha)=y^{\prime}(a)=0, \quad y(b)=y^{\prime}(b)=0,
$$

si trova, in base al teor. XIV, che: Tali equazioni possiedono l' unica soluzione nulla se esiste una funzione w sempre positiva in (a, b), con derivata terza. assolutamente continua e quarta generalmente continua, per la quale, in (a, b), riesca

$$
\begin{array}{ll}
\varphi_{0} \omega+\varphi_{1} \omega^{\prime}+\varphi_{2} \omega^{\prime \prime}+\varphi_{3} \omega^{\prime \prime \prime}-\omega^{\mathrm{r}} & \leq 0, \\
\varphi_{1} \omega+2 \varphi_{2} \omega^{\prime}+3 \varphi_{3} \omega^{\prime \prime}-4 \omega^{\prime \prime \prime} & =0, \\
\varphi_{2} \omega+3 \varphi_{3} \omega^{\prime}-6 \omega^{\prime \prime} & \geq 0,
\end{array}
$$

e quindi, in particolare, comunque risultino la costante a e le funzioni $\mathrm{F}(\mathrm{x})$ e $\mathrm{P}(\mathrm{x})$, generalmente continue e sommabili in $(\mathrm{a}, \mathrm{b}), \mathrm{P}(\mathrm{x})$ essendovi non negativa, se si può porre:

$$
\begin{aligned}
& \varphi_{3}=F \\
& \varphi_{2}=P+6 \alpha^{2}-3 x F \\
& \varphi_{1}=-8 \alpha^{3}+2 \alpha^{2} F-2 P \alpha
\end{aligned}
$$

soddisfacendo $\varphi_{0}$ la limitazione

$$
\varphi_{0} \leqq 3 \alpha^{4}-F \alpha^{3}+P \alpha^{2}
$$

L'osservaziene generale fatta' può dare frutti cospicui anche da un altro punto di vista, consentendo essa, come andiamo ad indicare, una genera: 
lizzazione dei teoremi fondamentali VII e VIII. Una soluzione $\left[y_{h}\right]$ del si* stema omogeneo $\left(27_{0}\right)$ sarà detta normale se tutte le sue componenti hanno nel punto iniziale a valori zero, meno una che ha valore uno. La trasformazione $y_{h}=\Sigma \omega_{h i} \eta_{k}$ trasforma soluzioni normali del sistema $\left(27_{0}\right)$ in soluzioni normali del sistema trasformato se la matrice della trasformazione è unitaria nel punto iniziale. Cio posto si può enunciare il teorema:

$\mathrm{XV}$. Se è possibile determinare gli elementi $\omega_{\mathrm{hk}}(\mathrm{x})$ della matrice della trasformazione $\mathrm{y}_{\mathrm{h}}=\Sigma \omega_{\mathrm{hk}} \gamma_{\mathrm{k}}$, in maniera che la matrice sia unitaria in a e diagonale in $\mathrm{b}$, e le funzioni

$$
\varphi{ }_{h k}^{*}=\sum_{\mu, \nu}^{1 . p} \varphi_{\mu, \nu} \Omega_{\mu h} \omega_{v k}-\sum_{\mu=1}^{p} \Omega_{\mu h} \omega_{\mu k}^{\prime}, \quad \text { per } \quad h \neq k, \quad(h, k=1, \ldots, p),
$$

siano non negative in $(\mathrm{a}, \mathrm{b})$, la componente $\mathrm{j}^{\mathrm{ma}}$ di una soluzione normale del sistema (27,) che sia diversa da zero in a è tale anche in $\mathrm{b}$, la componente $\mathrm{i}^{\mathrm{ma}}(\mathrm{i} \neq \mathrm{j})$ sarà pur essa diversa da zero in $\mathrm{b}$ se $\varphi_{\mathrm{ij}}{ }^{*}(\mathrm{x})$ non è identicamente nulla, oppure se per un certo indice $1 \neq \mathrm{i}$ è $\varphi_{1 \mathrm{j}}^{*}(\mathrm{x})$ non identicannente nulla ed essendo b un punto di continuità di $\varphi_{\mathrm{il}}^{*}(\mathrm{x})$ si ha $\varphi_{\mathrm{il}}{ }^{*}(\mathrm{~b})>0$.

L'applicazione di tale teorema sarà presumibilmente utile per un perfezionamento, nei singoli casi particolari, del teor. XIII, secondo il quale l'ipotesi $\varepsilon_{h k} \varphi_{h k}(x) \geqq 0$ in $(a, b)$, porta all'identico annullamento di molti coefficienti $\varphi_{k k}$.

3. Teoremi di confronto con condizioni terminali. - Facile conseguenza del teor. $V$ è questo:

XVI. Supposto l'intervallo (a, b) finito, le funzioni

$$
f\left(x, y_{1}, y_{2}\right), g\left(x, y_{1}, y_{2}\right)
$$

verifichino le ipotesi del teor. $\mathrm{V}$, per $\mathrm{p}=2$, e le $\mathrm{a}(\mathrm{x})$ e $\mathrm{v}(\mathrm{x})$ siano soluzioni, in $\mathrm{R}$, rispettivamente delle due equazioni differenziali del second'ordine:

$$
\begin{aligned}
& y^{\prime \prime}=f\left(x, y, y^{\prime}\right), \\
& y^{\prime \prime}=g\left(x, y, y^{\prime}\right),
\end{aligned}
$$

allora, dalle relazioni terminali

$$
u(a) \geqq v(a), \quad u(b) \geqq v(b),
$$

si deduce, in tutto (a, b),

e se

si $h a$

$$
\begin{aligned}
& u(x) \geqq v(x) . \\
& u(b)>v(b),
\end{aligned}
$$

Annali di Matematica, Serie IV. Tomo Xx. 
Ed invero, se per un punto $\xi$ di $(a, b)$ risultasse

$$
v \mid \xi)>u(\xi)
$$

esisterebbero dei punti $x_{1}$ e $x_{2}$ di $(a, b)$ per i quali si avrebbe

$$
\begin{gathered}
x_{1}<\xi<x_{2}, \\
u\left(x_{1}\right)=v\left(x_{1}\right), \quad u\left(x_{2}\right)=v\left(x_{2}\right),
\end{gathered}
$$

e, detto $x_{0}$ l' estremo superiore dei numeri $x_{1}$, si avrebbe

$$
\begin{gathered}
x_{0}<\xi \\
u\left(x_{0}\right)=v\left(x_{0}\right), \quad u^{\prime}\left(x_{0}\right) \leqq v^{\prime}\left(x_{n}\right),
\end{gathered}
$$

donde, per il teor. $\mathrm{V}$,

$$
v^{\prime}(x)-u^{\prime}(x) \geqq 0, \text { per } \quad x \geqq x_{n} .
$$

Ma

$$
\int_{x_{0}}^{x_{2}}\left(v^{\prime}(x)-u^{\prime}(x)\right) d x=\left[v\left(x_{2}\right)-u\left(x_{2}\right)\right]-\left[v\left(x_{11}\right)-u\left(x_{n}\right)\right]=0
$$

e quindi $v^{\prime}(x)-u^{\prime}(x) \equiv 0$ in $\left(x, x_{2}\right)$, e pertanto $v(\xi)=u(\xi)$, in contradizione con la (45). Dimostrata così la (42), se, essendo $u(b)>v(b)$, per un certo punto interno ad $(a, b)$, risultasse $v(\xi)=u(\xi)$, ivi si avrebbe anche $v^{\prime}(\xi)=u^{\prime}(\xi)$, e quindi, di nuovo in base al teor. V. $v(x) \geqq u(x)$, per $x \geq \xi$, donde $v(b) \geqq u(b)$, il che è assurdo.

Dal teor. VI si deduce il seguente:

XVII. Supposto l'intervallo (a, b) finito, le funzioni $\omega(\mathrm{x}), \varphi\left(\mathrm{x}, \mathrm{y}_{1}, \mathrm{y}_{2}\right)$, $\psi\left(\mathrm{x}, \mathrm{y}_{1}, \mathrm{y}_{2}\right)$ verifichino le ipotesi del teor. VI, per $\mathrm{p}=2$, e le $\mathrm{u}(\mathrm{y})$ e $\mathrm{v}(\mathrm{x})$ siano soluzioni, in $\mathrm{R}$, rispettivamente delle equazioni differenziali del second'ordine

$$
\begin{aligned}
& y^{\prime \prime}=\omega(x) \varphi\left(x, y, y^{\prime}\right) \\
& y^{\prime \prime}=\omega(x) \psi\left(x, y, y^{\prime}\right)
\end{aligned}
$$

allora dalle relazioni terminali (41) si deduce, in tutto (a, b), la (42), e, dalle (41) e (43), la (44) $\left({ }^{20}\right)$.

Se $\omega(\mathrm{x})=1$, ed essendo l'insieme $\mathrm{N}$, al piì, costituito dai punti a $e \mathrm{~b}$, per ogni punto interno all' intervallo (a, b), vale sempre una delle seguenti

(20) In questo teorema è contenuto, come particolarissimo caso, quello a cui ho fatto cenno nelle prime righe del presente scritto, mentre ciò non si può dire per i teoremi di confronto dati dalla Dott. IDA GRoppI nella nota: A proposito di alcuni criteri di confronto per le equazioni differeneiali del second" ordine. [ Bollettino dell' Unione matematica italiana ", Anno XVII (1938-XVI)], pp. 179-182. 
diseguaglianze

$\varphi\left(x, u(x), u^{\prime}(x)\right)<\psi\left(x, u(x), u^{\prime}(x)\right)$

$\varphi\left(x, v(x), v^{\prime}(x)\right)<\psi\left(x, v(x), v^{\prime}(x)\right)$,

dalle relazioni terminali (23) si deduce

$$
u(x)>v(x), \text { per } a<x<b .
$$

Dimostrata la prima parte del teorema, ciò che si ottiene con ragionamento identico a quello seguito per dimostrare il teor. XVI, se, nell'ipotesi della seconda parte, - per un punto $\xi$ interno ad $(a, b)$ risultasse $v(\xi)=u(\xi)$, ivi si avrebbe $v^{\prime}(\xi)=u^{\prime}(\xi)$, e quindi

$$
\begin{aligned}
v^{\prime \prime}(\xi)-u^{\prime \prime}(\xi) & =\psi\left(\xi, u(\xi), u^{\prime}(\xi)\right)-\varphi\left(\xi, u(\xi), u^{\prime}(\xi)\right), \\
& =\psi\left(\xi, v(\xi), v^{\prime}(\xi)\right)-\psi\left(\xi, v(\xi), v^{\prime}(\xi)\right),
\end{aligned}
$$

onde $v^{\prime \prime}(\xi)-u^{\prime \prime}(\xi)>0$, e pertanto $v(x)>u(x)$ in un intorno destro di $\xi$, il che è assurdo $\left({ }^{21}\right)$.

(21) Dunque, in particolare, se la funzione $\Psi\left(x, y_{1}, y_{2}\right)$ è, rispetto alla $x$, del tipo $C$ e generalmente continua nel dominio reltangolare $R\left[\left(a, a_{1}, a_{2}\right) ;\left(b . b_{1}, b_{2}\right)\right]$, e, comunque si fissino $x$ in $(a, b)$. fuori di un certo insieme $N$ privo di derivato, e $y_{2}$ nell'interno di $\left(a_{2}, b_{z}\right)$, funzione non decrescente di $y_{1}$, dal confronto delle due equazioni

$$
\begin{aligned}
& y^{\prime \prime}=0 \\
& y^{\prime \prime}=(n)(x) \downarrow\left(x, y, y^{\prime}\right)
\end{aligned}
$$

si ricava ché: se la seconda ¿̀ unitaria nella sua soluzione $v(x)$, in $R$, ed esistono due co* stanti $c_{1}$ e $c_{2}$, per le quali si ha. in $(a, b)$,

e generalmente

$$
a_{1}<c_{1} \frac{b-x}{b-a}+c_{2} \frac{x-a}{b-a}<b_{1}, \quad a_{2}<\frac{c_{2}-c_{1}}{b-a}<b_{2}
$$

$$
\psi\left(x, c_{1} \frac{b-x}{b-a}+c_{2} \frac{x-a}{b-a}, \quad \frac{c_{2}-c_{1}}{b-a}\right) \geqq 0,
$$

allora dalle relazioni terminali

$(\alpha)$

segue, in tutto $(a, b)$,

(B)

$$
v(a) \leqq c_{1}, \quad v(b)<c_{2},
$$

$$
v(x) \leqq c_{1} \frac{b-x}{b-a}+c_{2} \frac{x-a}{b-a} .
$$

Se, per esempio, assegnata l'equazione lineare

$$
y^{\prime \prime}=\psi(x)+\psi_{0}(x) y+\psi_{1}(x) y^{\prime}
$$

con $\psi, \psi_{0}, \psi_{1}$ generalmente continue in $(a, b)$, e ivi sommabili, si ha. in $(a, b)$, generalmente,

$$
\begin{aligned}
& \psi_{0}(x) \geqq 0, \\
&(b-a)\rfloor(x)+\left[c_{1}(b-x)+c_{z}(x-a)\right] \psi_{0}(x)+\left(c_{2}-c_{1}\right) \psi_{1}(x) \geq 0,
\end{aligned}
$$

per ogni soluzione $v(x)$ dell' equazione, verifieante le relazioni terminali $(\alpha)$, si ha la limitazione ( $\beta$ ). Si ritrova cosi un ben noto risultato della teoria delle equazioni differenziali lineari del second'ordine, di solito enunciato per $c_{1}=c_{2}=0$. 
4. Teoremi d'esistenza per le soluzioni dei sistemi di equazioni differenziali ordinarie, dipendenti da un parametro, in grande nel parametro. Dal teor. I di Caratheodony e dai teoremi II e III si deduce facilmente il seguente:

XVIII. Nel dominio rettangolare di punti estremi inferiore $\left(a, a_{1}, \ldots, a_{p}, \lambda^{\prime}\right)$ e superiore $\left(b, b_{1}, \ldots, b_{p}, \lambda^{\prime \prime}\right)$, dello spazio $\left(x, y_{1}, \ldots, y_{p}, \lambda\right)$, le funzioni

$$
\alpha_{h}(\lambda), \quad f_{h}\left(x, y_{1}, \ldots, y_{p}, \lambda\right), \quad(h=1, \ldots, p),
$$

siano di tipo $\mathrm{C}$, rispetto alla $\mathrm{x}$, e, avendosi

$$
a_{h}<\alpha_{h_{k}}(\lambda)<b_{k}, \quad\left(h=1, \ldots, p, \lambda^{\prime}<\lambda<\lambda^{\prime \prime}\right),
$$

e supposto l'intervallo $(\mathrm{a}, \mathrm{b})$ finito, per due valori $\lambda_{1}$ e $\lambda_{2}$ di $\lambda$, interni all'intervallo $\left(\lambda^{\prime}, \lambda^{\prime \prime}\right)$, con $\lambda_{1}<\lambda_{2}$, le $\left[\mathrm{u}_{\mathrm{h}}(\mathrm{x})\right]$ e $\left[\mathrm{v}_{\mathrm{h}}(\mathrm{x})\right]$ siano soluzioni, nel dominio rettangolare $\mathrm{R}\left[\left(\mathrm{a}, \mathrm{a}_{1}, \ldots, \mathrm{a}_{\mathrm{p}}\right),\left(\mathrm{b}, \mathrm{b}_{1}, \ldots, \mathrm{b}_{\mathrm{p}}\right)\right]$, dei sistemi di equazioni differenziali e di condizioni iniziali che si ottengono dal sistema

$$
\left\{\begin{array}{l}
y_{k}{ }^{\prime}=f_{h}\left(x, y_{1}, \ldots, y_{p}, \lambda\right), \\
y_{h}(a)=\alpha_{h}(\lambda),
\end{array} \quad(h=1, \ldots, p),\right.
$$

ponendovi, rispettivamente, $\lambda=\lambda_{1}$ e $\lambda=\lambda_{2}$, il sistema di equazioni differenziali conservandosi unitario, in $\mathrm{R}$, per ogni valore di $\lambda$ nell' intervallo (chiuso) $\left(\lambda_{1}, \lambda_{z}\right)$. In ciascuna delle seguenti ipotesi:

d) mantenendo $\lambda$ nell' intervallo $\left(\lambda_{1}, \lambda_{2}\right)$, comunque si fissi $\mathrm{x}$ in $(\mathrm{a}, \mathrm{b})$, fuori di un certo insieme di misura nulla, le $\mathrm{f}_{\mathrm{h}} e \alpha_{\mathrm{h}}$ sono funzioni non decrescenti delle rimanenti variabili;

$\left.\mathrm{d}^{\prime \prime}\right) \grave{e}$

$$
f_{h} \equiv \omega_{h}(x) \varphi_{h}\left(x, y_{1}, \ldots, y_{p}, \lambda\right), \quad(h=1, \ldots, p),
$$

con $\omega_{\mathrm{h}}(\mathrm{x})$ sommabile in $(\mathrm{a}, \mathrm{b})$, ivi quasi ovunque non negative, $\varphi_{\mathrm{h}}$ generalmente continue, rispetto alla $\mathrm{x}$, e, mantenendo $\lambda$ nell' intervallo $\left(\lambda_{1}, \lambda_{2}\right)$, comunque si fissino $\mathrm{x}$ in (a, b), fuori di un certo insieme privo di derivate, $e \mathrm{y}_{\mathrm{h}}$ nell' in. terno dell' intervallo $\left(\mathrm{a}_{\mathrm{h}}, \mathrm{b}_{\mathrm{h}}\right)$, le $\varphi_{\mathrm{h}} e \alpha_{\mathrm{h}}$ sono funzioni non decrescenti di ciascuna delle rimanenti variabili,

si può costruire un intervallo $\left(\lambda_{1}{ }^{\prime}, \lambda_{2}{ }^{\prime \prime}\right)$, al quale l'intervallo $\left(\lambda_{1}, \lambda_{2}\right) \grave{e}$ interno, contenuto nell'intervallo $\left(\lambda^{\prime}, \lambda^{\prime \prime}\right)$ e, per $\lambda$ in $\left(\lambda_{1}^{\prime}, \lambda_{2}^{\prime \prime}\right)$ e $\mathrm{x}$ in $(\mathrm{a}, \mathrm{b})$, definire una soluzione $\left[\mathrm{y}_{\mathrm{h}}(\mathrm{x}, \lambda)\right]$, in $\mathrm{R}$, del sistema (46). Per $\lambda$ in $\left(\lambda_{1}, \lambda_{2}\right)$ le $\mathrm{y}_{\mathrm{h}}(\mathrm{x}, \lambda)$ sono funzioni continue del punto $(\mathrm{x}, \lambda)$, per ogni fissata $\mathrm{x}$ non decrescente in $\lambda$, e verificano le limitazioni

$$
u_{h}(x) \leqq y_{h}(x, \lambda) \leqq v_{h}(x), \quad(h=1, \ldots, p) .
$$

Ci limiteremo a dimostrare il teorema nell'ipotesi $d)$, nella quale, dunque, si può fissare, in $(a, b)$, un insieme $N$, di misura nulla, tale che per ogni 
punto $x$ di $(a, b)$, fuori di esso, le $f_{k}$ siano funzioni continue del punto $\left(y_{1}, y_{2}, \ldots, y_{p}, \lambda\right)$, non decrescenti in ciascuna delle variabili $y_{1}, y_{2}, \ldots, y_{n}, \lambda$, e si ha:

$$
\left|f_{h}\left(x, y_{1}, \ldots, y_{p}, \lambda\right)\right| \leqq F(x),
$$

con $F(x)$ sommabile in $(a, b)$. Indicheremo con $I$ l'insieme di punti che si ottiene dall'intervallo $(a, b)$ privandolo dei punti di $N$. Poichè, per $x$ in $I$,

$$
\begin{gathered}
f_{k}\left(x, u_{1}(x), \ldots, u_{p}(x), \lambda_{1}\right) \leqq f_{k}\left(x, u_{1}(x), \ldots, u_{p}(x), \lambda_{2}\right), \\
\alpha_{k}\left(\lambda_{1}\right) \leqq \alpha_{h}\left(\lambda_{2}\right),
\end{gathered}
$$

possiamo, intanto, in base al teor. II, asserire che, in $(a, b)$,

$$
u_{h}(x) \leqq v_{h}(x) \text {. }
$$

In virtù del teor. I si può costruire un intorno $\left(\lambda_{1}-\sigma^{\prime}, \lambda_{1}+\sigma^{\prime \prime}\right)$ del punto $\lambda_{1} \mathrm{e}$, per $x$ in $(a, b)$ e $\lambda$ in tale intorno, definire una soluzione $\left[y_{h}(x, \lambda)\right]$, in $R$, del sistema (46). Per $x$ in $I$ e per ogni valore $\lambda$ dell'intorno destro $\left(\lambda_{1}, \lambda_{1}+\sigma^{\prime \prime}\right)$ di $\lambda_{1}$, che non superi $\lambda_{2}$, si ha:

$$
\begin{gathered}
f_{h}\left(x, u_{1}(x), \ldots, u_{p}(x), \lambda_{1}\right) \leqq f_{h}\left(x, u_{1}(x), \ldots, u_{p}(x), \lambda\right), \\
f_{h}\left(x, y_{1}(x, \lambda), \ldots, y_{p}(x, \lambda), \lambda\right) \leqq f_{h}\left(x, y_{1}(x, \lambda), \ldots, y_{p}(x, \lambda), \lambda_{2}\right),
\end{gathered}
$$

e quindi, in $(a, b)$,

$$
u_{h}(x) \leqq y_{h}(x, \lambda) \leqq v_{h}(x), \quad\left(\text { per } \quad \lambda_{1} \leqq \lambda \leqq \lambda_{1}+\sigma^{\prime \prime} \quad, \quad h=1, \ldots, p\right)
$$

Esistono dunque dei valori $\mu$ dell' intervallo $\left(\lambda_{1}, \lambda_{2}\right)$, maggiori di $\lambda_{1}$, tali che per ogni $\lambda$ dell'intervallo (chiuso) $\left(\lambda_{1}, \mu\right)$ si può definire una soluzione $\left[y_{h}(x, \lambda)\right]$, in $R$, del sistema $(46)$, verificante le limitazioni $(47)$. Detto $\mu_{0}$ l'estremo superiore dell'insieme numerico descritta da tali $\mu$, sarà $\mu_{0} \leqq \lambda_{z}$, e il teorema può ritenersi completamente dimostrato se faremo vedere che, precisamente, riesce $\mu_{0}=\lambda_{z}$. Costruiremo, a tale scopo, una successione crescente $\mu_{1}, \mu_{2}, \ldots, \mu_{\eta}, \ldots$, di valori di $\mu$ avente per limite $\mu_{0}$. Per $x$ in $I$ si ha:

$$
\begin{gathered}
f_{h}\left(x, y_{1}\left(x, \mu_{1}\right), \ldots, y_{p}\left(x, \mu_{n}\right), \mu_{n}\right) \leqq f_{h_{k}}\left(x, y_{1}\left(x, \mu_{n}\right), \ldots, y_{p}\left(x, \mu_{n}\right), \mu_{n+1}\right), \\
f_{h}\left(x, u_{1}(x), \ldots, u_{p}(x), \lambda_{1}\right) \leqq f_{h}\left(x, u_{1}(x), \ldots, u_{p}(x), \mu_{n}\right), \\
f_{h}\left(x, y_{1}\left(x, \mu_{1}\right), \ldots, y_{p}\left(x, \mu_{n}\right), \mu_{n}\right) \leqq f_{k}\left(x, y_{1}\left(x, \mu_{n}\right), \ldots, y_{p}\left(x, \mu_{n}\right), \lambda_{2}\right),
\end{gathered}
$$

e quindi, per $x$ in $(a, b)$,

$$
u_{h}(x) \leqq y_{h}\left(x, \mu_{n}\right) \leqq y_{k}\left(x, \mu_{n+1}\right) \leqq v_{h}(x) .
$$

Esiste, pertanto, per ogni $x$ in $(a, b)$, il limite

$$
\lim _{n \rightarrow \infty} y_{k}\left(x, \mu_{n}\right) \text {, }
$$

e, indicandolo con $y_{k}^{\prime \prime}(x)$, si ha

$$
a_{h}<u_{h}(x) \leqq y_{h}{ }^{\prime \prime}(x) \leqq v_{h}(x)<b_{h} .
$$


Per ogni $x$ in $I$ si ha

$$
\begin{gathered}
\lim _{n \rightarrow \infty} f_{b}\left(x, y_{1}\left(x, \mu_{n}\right), \ldots, y_{p}\left(x, \mu_{n}\right), \mu_{n}\right)=f_{k}\left(x, y_{k}{ }^{\circ}(x), \ldots, y_{p}{ }^{n}(x), \mu_{0}\right) \\
\left|f_{h}\left(x, y_{1}\left(x, \mu_{n}\right), \ldots, y_{p}\left(x, \mu_{n}\right), \mu_{n}\right)\right| \leqq F(x),
\end{gathered}
$$

donde la sommabilità in $|a, b|$, della funzione

$$
f_{h}\left(x, y_{k}{ }^{0}(x), \ldots, y_{k}{ }^{0}(x), \mu_{0}\right)
$$

laddove, per essere $\lim x_{h}\left(\mu_{n}\right)$ (per $\left.n \rightarrow \infty\right)=\alpha_{h}\left(\mu_{n}\right)$, dall' equazione

$$
y_{h}\left(x, \mu_{n}\right)=\alpha_{h}\left(\mu_{n}\right)+\left.\right|_{a} ^{\infty} f_{h}\left(t, y_{i}\left(t, \mu_{n}\right), \ldots, y_{p}\left(t, \mu_{n}\right), \mu_{n}\right) d t,
$$

passando al limite per $n \rightarrow \infty$, si ricava, per $x$ in $(a, b)$,

$$
y_{i}{ }^{0}(x)=\alpha_{h}\left(\mu_{0}\right)+\int_{a}^{i} f_{i}\left(t, y_{1}{ }^{0}(t), \ldots, y_{p}{ }^{0}(t), \mu_{0}\right) d t .
$$

Si ha dunque che per $\lambda=\mu_{0}$ esiste la soluzione $\left[y_{k}{ }^{0}(x)\right]$, in $R$, del sistema (46), con le $y_{t}^{\prime \prime}(x)$ verificanti le limitazioni (29). Se fosse $\mu_{0}<\lambda_{2}$, si potrebbero trovare, in base al teor. I, valori di $\mu>\mu_{11}$, dell' intervallo $\left(\lambda_{1}, \lambda_{2}\right)$, tali che per ogni $\lambda$ dell'intervallo $(\lambda, \mu)$ si potrebbe costruire una soluzione $\left[y_{i}(x, \lambda)\right]$, in $R$, del sistema $(46)$, con le $y_{h}(x, \lambda)$ verificanti le $(47)$, il che è assurdo, per essere $\mu_{0}$ l'estremo superiore dell'insieme numerico costitaito dai detti valori.

Un teorema analogo, che tralasciamo di enunciare, sussiste. ovviamente, per le equazioni

$$
\begin{aligned}
& y^{(p)}=f\left(x, y, y^{\prime}, \ldots, y^{(p-1)}, \lambda\right), \\
& y^{(k)}(a)=\alpha_{\bar{k}}(\lambda),
\end{aligned}
$$

dipendenti dal parametro $\lambda$. Rileviamo di esso un caso particolare interessante. Sia $\varphi\left(x, y_{1}, \ldots y_{p}\right)$, rispetto alla $x$, di tipo $C$ nel dominio rettangolare $R\left[\left(a, a_{t}, \ldots, a_{p}\right) ;\left(b, b_{1}, \ldots, b_{p}\right)\right]$ e generalmente continua e verifichi la condizione di LtPSOHITz, secondo CARATHEODORY, si abbia cioè, per quasi tutti i valori di $x$ in $(a, b)$,

$$
\left|\varphi\left(x, u_{1}, \ldots, u_{p}\right)-\varphi\left(x, v_{1}, \ldots, v_{p}\right)\right| \leqq \dot{L}(x) \sum_{h=1}^{p}\left|u_{h}-v_{h}\right|,
$$

comunque si scelgano le quantità $u_{h}$ e $v_{h}$ nell' intervallo $\left(a_{h}, b_{h}\right)(h=1, \ldots, p)$, essendo $L(x)$ una funzione sommabile in $(a, b)$. L' equazione differenziale

$$
y^{(p)}=\lambda \varphi\left(x \cdot y, y^{\prime}, \ldots \cdot y^{(p-1)}\right)
$$


riesce allora unitaria, in $R$, qualunque valore si dia al parametro $\lambda$. Sia $u(x)$ una soluzione. in $R$, dell' equazione

e si abbia

$$
y^{(p)}=p\left(x, y, y^{\prime}, \ldots, y^{(p-1)}\right),
$$

$$
\begin{array}{cr}
u^{(k)}(a)=\alpha_{k} . & (k=0,1, \ldots, p-1), \\
\alpha_{k} \geqq 0, & (k=0,1, \ldots, p-1), \\
a_{k}<0<b_{h}, & (h=1,2, \ldots, p),
\end{array}
$$

e, in $R$, generalmente rispetto alla $x$,

$$
\varphi\left(x, y_{1}, \ldots, y_{p}\right) \geq 0
$$

e non decrescente in ciascuna delle $p-1$ variabili $y_{1}, \ldots, y_{p-1}$. In tali ipotesi si può affermare, in base al teor. IX, l'esistenza di un intervallo $\left(\lambda^{\prime}, \lambda^{\prime \prime}\right)$, con $\lambda^{\prime}<0$ e $\lambda^{\prime \prime}>1$, tale che per ogni valore di $\lambda$ in tale intervallo è possibile definire una soluzione $y(x, \lambda)$, in $R$, delle equazioni:

$$
\begin{aligned}
y^{(p)}= & \lambda \varphi\left(x, y, y^{\prime}, \ldots, y^{(p-1)}\right), \\
& y^{(k)}(a)=\lambda \alpha_{k},
\end{aligned} \quad(k=0,1, \ldots, p-1),
$$

risultando, per $\lambda$ nell'intervallo $\{0,1)$,

$$
\left.0 \leqq y^{(k)}(x, \lambda) \leqq u^{(k)}(x)\right), \quad(k=0,1, \ldots, p-1),
$$

e le $y^{(k)}(x, \lambda)(k=0.1, \ldots, p-1)$, per ogni fissato $x$, funzioni non decrescenti di $\lambda$.

Non si pensi che tale intervallo $\left(\lambda^{\prime}, \lambda^{\prime \prime}\right)$, di variabilità del parametro $\lambda$, consentita dal teorema, riesca, in generale, banalmente ristretto. $\grave{E}$ istruttivo, al riguardo, l'esempio delle semplieissime equazioni :

$$
\begin{aligned}
y^{\prime} & =\lambda y^{2}, \\
y(0) & =\lambda,
\end{aligned}
$$

considerate al variare di $x$ nell'intervallo $(0, b)$, con $b<1$, la cui solnzione

$$
y=\frac{\lambda}{1-\lambda^{2} x},
$$

può definirsi nell' intervallo $(0, b)$ solo se $\lambda$ si mantiene in un intervallo $\left(\lambda^{\prime}, \lambda^{\prime \prime}\right)$ interno all'intervallo $(-1 / \sqrt{b}, 1 / \sqrt{b})$, fra i quali vi è bene l'intervallo $(0,1)$, il cai estremo destro può essere però vicino tanto quanto si vuole a $1 / \mathrm{V} \bar{b}$, pur di prendere $b$ abbastanza prossimo a uno. 